\title{
Global Existence and Exponential Decay of Strong Solutions to the 2D Density-dependent Nematic Liquid Crystal Flows with Vacuum
}

\author{
Yang Liu
}

\begin{abstract}
This paper deals with the 2D incompressible nematic liquid crystal flows with density-dependent viscosity in bounded domain. The global well-posedness of strong solutions are established in the vacuum cases, provided the assumption that $\bar{\rho}+\left\|\nabla d_{0}\right\|_{L^{2}}$ is suitably small with large velocity, which extends the recent work [Discrete Contin. Dyn. Syst. 37 (2017), 4907-4922] and [Methods Appl. Anal. 22 (2015), 201-220] to the case of variable viscosity. Furthermore, the exponential decay of the solution is also obtained.
\end{abstract}

\section{Introduction}

The motion of incompressible nematic liquid crystal flows, which are described by the following simplified version of the Ericksen-Leslie equations:

$$
\left\{\begin{array}{l}
\rho_{t}+\operatorname{div}(\rho u)=0, \\
\rho u_{t}+\rho(u \cdot \nabla) u-\operatorname{div}(\mu(\rho) \nabla u)+\nabla P=-\lambda \operatorname{div}(\nabla d \odot \nabla d), \\
\operatorname{div} u=0, \quad|d|=1 \\
d_{t}+u \cdot \nabla d=\theta\left(\Delta d+|\nabla d|^{2} d\right),
\end{array}\right.
$$

in $\Omega \times[0, \infty)$, where $\Omega$ is a bounded domain with smooth boundary in $\mathbb{R}^{2}$. Here $u: \Omega \times$ $[0, \infty) \rightarrow \mathbb{R}^{2}$ represents the velocity field of the flow, $d: \Omega \times[0, \infty) \rightarrow \mathcal{S}^{2}$, the unit sphere in $\mathbb{R}^{2}$, represents the macroscopic molecular orientation of the liquid crystal material, $\rho$ and $P$ are scalar functions, respectively, denoting the density of the fluid and the pressure arising from the usual assumption of incompressibility $\operatorname{div} u=0$. The positive constants $\lambda$ and $\theta$ represent viscosity of fluid, competition between kinetic and potential energy, and microscopic elastic relaxation time respectively. The viscosity coefficient $\mu=\mu(\rho)$ is a general function of density, which is assumed to satisfy

$$
\mu \in C^{1}[0, \infty) \text { and } \quad 0<\underline{\mu} \leq \mu \leq \bar{\mu}<\infty \quad \text { on }[0, \infty)
$$

Received September 8, 2019; Accepted February 25, 2020.

Communicated by Tai-Peng Tsai.

2010 Mathematics Subject Classification. 76A15, 35B65, 35Q35.

Key words and phrases. incompressible nematic liquid crystal flow, strong solutions, vacuum, exponential decay. 
for some positive constant $\underline{\mu}$ and $\bar{\mu}$. Without loss of generality, both $\lambda$ and $\theta$ are normalized to 1 . The symbol $\nabla d \odot \nabla d$, which exhibits the property of the anisotropy of the material, denotes the $n \times n$ matrix whose $(i, j)$-th entry is given by $\partial_{i} d \cdot \partial_{j} d$, for $i, j=1,2$.

Noticing that

$$
\operatorname{div}(\nabla d \odot \nabla d)=\Delta d \cdot \nabla d+\nabla\left(\frac{|\nabla d|^{2}}{2}\right),
$$

one can rewrite equation $(1.1)_{2}$ as

$$
\rho u_{t}+\rho(u \cdot \nabla) u-\operatorname{div}(\mu(\rho) \nabla u)+\nabla P=-\lambda \Delta d \cdot \nabla d .
$$

We consider an initial boundary value problem for (1.1) with the following initial and boundary conditions:

$$
\left.(\rho, \rho u, d)\right|_{t=0}=\left(\rho_{0}, \rho_{0} u_{0}, d_{0}\right), \quad\left|d_{0}\right|=1 \quad \text { in } \Omega ; \quad u=0, \quad d=d_{0}^{\prime} \quad \text { on } \partial \Omega
$$

with $d_{0}^{\prime} \in \mathcal{S}^{2}$ being given with compatibility, $\operatorname{div} u_{0}=0$ in $\Omega$ and $d_{0}^{\prime} \in C^{1}(\bar{\Omega})$ satisfying $\nabla d_{0}^{\prime}=0$ on the boundary $\partial \Omega$ (see e.g., $[7]$ ).

The hydrodynamics of nematic liquid crystals developed by Ericksen [2] and Leslie [12] in the 1960's, but it still retains most important mathematical structures as well as most of the essential difficulties of the original Ericksen-Leslie model. Mathematically, system (1.1) is a strongly coupled system between the nonhomogeneous incompressible Navier-Stokes equations and the transported heat flows of harmonic map, and thus, its mathematical analysis is full of challenges.

When the fluid is the homogeneous case, the systems (1.1) are the simplified model of nematic liquid crystals with constant density. Huang-Wang [9] have provided a blow-up criterion of strong solutions, and the well-posedness of (1.1) for an initial data $\left(u_{0}, d_{0}\right)$ with small $\mathrm{BMO}^{-1} \times \mathrm{BMO}^{-n o r m}$, and with small $L_{\text {uloc }}^{3}\left(\mathbb{R}^{3}\right)$-norm has been shown by Wang 25 and Hineman-Wang [5], and small $L^{3}\left(\mathbb{R}_{+}^{3}\right)$-norm has been established by Huang-WangWen [8], respectively. Li [13 proved the local well-posedness of mild solutions with $L^{\infty}$ initial data, in particular, that the initial energy may be infinite. Most recently, LinWang [20] have shown the existence of global weak solutions in dimension three under the assumption that the initial director field $d_{0} \subset \mathbb{S}_{+}^{2}$.

Let's go back to the system (1.1). When viscosity $\mu$ is a constant, there is a huge literature on the studies about well-posedness of solutions. In the absence of vacuum, Li [14 and Wen-Ding [26] established the global strong solutions in dimension two. For small data, Li-Wang [19 obtained global strong solutions in three dimensional space. On the other hand, when the initial density allows to vanish, if the initial data are small (in some sense) and satisfy the additional compatibility condition, Li [15], Yu-Zhang [27] and Ding-Huang-Xia [1] obtained the global existence of strong solutions in dimension three. Li 18 considered the case on the bounded domains in $\mathbb{R}^{2}$. Recently, Liu-Zhang 23 and 
Liu et al. 22 independently extended the result of 18 to $\mathbb{R}^{2}$ with large initial data, provided that the initial orientation $d_{0}=\left(d_{01}, d_{02}, d_{03}\right)$ satisfies a geometric condition

$$
d_{03} \geq \epsilon_{0} \text { for some positive } \epsilon_{0}>0 \text {. }
$$

Li-Liu-Zhong [17 got the same result under small initial data without the additional geometric condition (1.5). Recently, when the viscosity coefficient is a function of the density of fluid, if the initial data satisfies the following compatibility condition

$$
-\operatorname{div}\left(\mu\left(\rho_{0}\right) \nabla u_{0}\right)+\nabla P_{0}+\operatorname{div}\left(\nabla d_{0} \odot \nabla d_{0}\right)=\sqrt{\rho_{0}} g \quad \text { for some }\left(\nabla P_{0}, g\right) \in L^{2},
$$

Gao-Tao-Yao [3 established the local unique strong solutions to the system (1.1] in a bounded domain of $\mathbb{R}^{N}(N=2,3)$ with the Neumann boundary condition, and they also obtained the Serrin-type blow up criterion of the strong solutions.

Especially, Li [18 (also see [15]) studied global regularity for $d=d_{0}^{\prime}$ on $\partial \Omega$ when initial energy is small. However, when deal with the case of variable viscosity, some new difficulties need to be overcome and the similar result is still unclear. Our main aim in this paper is to offer an answer to this open problem. Indeed, we will establish the global existence of strong solutions to the 2D incompressible nematic liquid crystal system (1.1) (1.4), under the assumption that the initial data in some norm is small enough, and we don't need any smallness on the initial velocity.

Before stating the main results, we first explain the notations and conventions used throughout this paper. Set

$$
\int f d x:=\int_{\Omega} f d x
$$

For $1 \leq r \leq \infty$ and $k \in \mathbb{N}$, the Sobolev spaces are defined in a standard way,

$$
L^{r}:=L^{r}(\Omega), \quad W^{k, r}:=\left\{f \in L^{r}: D^{\alpha} f \in L^{r},|\alpha| \leq k\right\}, \quad H^{k}:=W^{k, 2} .
$$

Moreover, $H_{0}^{1}$ and $H_{0, \sigma}^{1}$ represent the closure of $C_{0}^{\infty}$ and $C_{0, \sigma}^{\infty}:=\left\{f \in C_{0}^{\infty}: \operatorname{div} f=0\right\}$ in $H^{1}$, respectively.

Our main result can be stated as follows:

Theorem 1.1. Let $\Omega$ be a bounded smooth domain in $\mathbb{R}^{2}$ and $q \in(2, \infty)$ be a fixed constant. For any given positive numbers $M_{0}, M_{1}, M_{2}$, and $M_{3}$, suppose that the initial data $\left(\rho_{0}, \rho_{0} u_{0}, d_{0}\right)$ satisfies the regularity conditions

$$
0 \leq \rho_{0} \leq \bar{\rho}, \quad \rho_{0} \in H^{1} \cap W^{1, q}, \quad \nabla \mu\left(\rho_{0}\right) \in L^{q}, \quad u_{0} \in H_{0, \sigma}^{1}, \quad d_{0} \in H^{2}, \quad\left|d_{0}\right|=1 .
$$

Then for

$$
\begin{aligned}
\bar{\rho}+\left\|\nabla d_{0}\right\|_{L^{2}} & =: M_{0}, \quad\left\|\sqrt{\rho_{0}} u_{0}\right\|_{L^{2}}^{2}+\left\|\nabla d_{0}\right\|_{L^{2}}^{2}=: M_{2}, \\
\left\|\nabla \mu\left(\rho_{0}\right)\right\|_{L^{q}} & =: M_{1}, \quad \bar{\mu}\left\|\nabla u_{0}\right\|_{L^{2}}^{2}+\left\|\Delta d_{0}\right\|_{L^{2}}^{2}=: M_{3},
\end{aligned}
$$


there exists some small positive constant $\epsilon_{0}$ depending on $\Omega, \underline{\mu}, \bar{\mu}, M_{1}, M_{2}$, and $M_{3}$ such that if

$$
M_{0} \leq \epsilon_{0},
$$

then the initial boundary value problem (1.1)-(1.4) admits a unique global strong solution $(\rho, u, d, P)$ satisfying that any $0<\tau<T<\infty$ for any $r$ with $2<r<\min \{q, 3\}$,

$$
\left\{\begin{array}{l}
0 \leq \rho \in C\left([0, T] ; H^{1} \cap W^{1, q}\right), \\
\nabla u \in L^{\infty}\left(0, T ; L^{2}\right) \cap L^{\infty}\left(\tau, T ; H^{1}\right) \cap L^{2}\left(\tau, T ; W^{1, r}\right), \\
P \in L^{\infty}\left(0, T ; L^{2}\right) \cap L^{\infty}\left(\tau, T ; H^{1}\right) \cap L^{2}\left(\tau, T ; W^{1, r}\right), \\
d \in L^{\infty}\left(0, T ; H^{2}\right) \cap L^{\infty}\left(\tau, T ; H^{3}\right) \cap L^{2}\left(\tau, T ; H^{4}\right), \\
d_{t} \in L^{\infty}\left(\tau, T ; H^{1}\right) \cap L^{2}\left(\tau, T ; H^{2}\right), \\
\nabla \mu(\rho) \in C\left([0, T] ; L^{q}\right), \quad|d|=1 \quad \text { in } \Omega \times[0, T] .
\end{array}\right.
$$

Moreover, it holds that

$$
\sup _{0 \leq t<\infty}\|\nabla \rho\|_{L^{2} \cap L^{q}} \leq 2\left\|\nabla \rho_{0}\right\|_{L^{2} \cap L^{q}}, \quad \sup _{0 \leq t<\infty}\|\nabla \mu(\rho)\|_{L^{q}} \leq 2\left\|\nabla \mu\left(\rho_{0}\right)\right\|_{L^{q}},
$$

and that there exists some positive constant $\sigma$ depending only on the constant of Poincaré's inequality, $\Omega, \bar{\rho}$, and $\underline{\mu}$ such that for all $t \geq 1$,

$$
\left\|\nabla d_{t}\right\|_{L^{2}}^{2}+\|\nabla d\|_{H^{2}}^{2}+\|\nabla u(\cdot, t)\|_{H^{1}}^{2}+\|P(\cdot, t)\|_{H^{1}}^{2} \leq C e^{-\sigma t},
$$

where $C$ depends only on $\Omega, q, \underline{\mu}, \bar{\mu}, M_{1}, M_{2}$, and $M_{3}$.

Remark 1.2. Theorem 1.1 extends the result of $\mathrm{Li}[18$ (also see [15]) to the case of variable viscosity for arbitrarily large initial velocity with a smallness assumption only on $\bar{\rho}+$ $\left\|\nabla d_{0}\right\|_{L^{2}}$, which is in sharp contrast to [15, 18] where they need the smallness assumption on the initial energy.

Remark 1.3. Compared with 15,18 , there is no need to impose the additional compatibility conditions on the initial data for the global existence of the strong solutions. Furthermore, it should be pointed out that the exponential decay of the global strong solution in $(1.9)$ is completely new for the $2 \mathrm{D}$ nonhomogeneous nematic liquid crystal flows.

Remark 1.4. When $d$ is a constant vector and $|d|=1$, system (1.1) turns to be the nonhomogeneous incompressible Navier-Stokes equations. Theorem 1.1 is different from the result of Huang-Wang [11], since they need the smallness assumptions on $\left\|\nabla \mu\left(\rho_{0}\right)\right\|_{L^{q}}$. Moveover, Theorem 1.1 established the exponential decay-in-time properties, since the corresponding decay-in-time rates of $[11]$ for the strong solutions to $(1.1)-(1.4)$ is algebraic. 
We now make some comments on the analysis in this paper. To extend the local strong solutions whose existence is obtained by Lemma 2.1 globally in time, one needs to establish global a priori estimates on smooth solutions to (1.1)-(1.4) in suitable higher norm. The key ingredient here is to get the time-independent bounds on the $L^{1}\left(0, T ; L^{\infty}\right)$-norm of $\nabla u$ and then the $L^{\infty}\left(0, T ; L^{q}\right)$-norm of $\nabla \mu(\rho)$ and the $L^{\infty}\left(0, T ; L^{2}\right)$-one of $\nabla \rho$. Here, some key ideas due to [11] will be used, which deals with the $2 \mathrm{D}$ incompressible Navier-Stokes. It should be pointed out that the crucial techniques of proof in [11] cannot be adapted directly to the situation treated here. On the one hand, as mentioned by Huang-Wang [11], the methods used in [11] depend crucially on the smallness assumptions on $\left\|\nabla \mu\left(\rho_{0}\right)\right\|_{L^{q}}$ and the following inequality

$$
\|\sqrt{\rho} u\|_{L^{4}}^{2} \leq C(\bar{\rho}, \Omega)\left(1+\|\rho u\|_{L^{2}}\right)\|\nabla u\|_{L^{2}} \sqrt{\log \left(2+\|\nabla u\|_{L^{2}}^{2}\right)} .
$$

On the other hand, compared with [11], for system (1.1)-(1.4) treated here, the strong coupling terms and strong nonlinear terms, such as $u \cdot \nabla d, \operatorname{div}(\nabla d \odot \nabla d)$, and $|\nabla d|^{2} d$ will bring out some new difficulties. Hence, some new ideas are needed here. First, using the initial layer analysis (see $[4,6,10]$ ), we succeed in bounding the $L^{1}\left(0, \min \{1, T\} ; L^{\infty}\right)$ norm of $\nabla u$ by $\bar{\rho}$ and $\sup _{0 \leq t \leq T}\|\nabla d\|_{L^{2}}$ (see (3.34). Then, in order to estimate the $L^{1}\left(\min \{1, T\}, T ; L^{\infty}\right)$-norm of $\nabla u$, we find that $\|\sqrt{\rho} u\|_{L^{2}}^{2}+\|\nabla d\|_{L^{2}}^{2}$ in fact decays at the rate of $e^{-\sigma t}(\sigma>0)$ for large time (see $(3.5)$ ), which can be achieved by combining the standard energy equality (see (3.7)) with the following fact

$$
\|\sqrt{\rho} u\|_{L^{2}}^{2}+\|\nabla d\|_{L^{2}}^{2} \leq\|\rho\|_{L^{\infty}}\|u\|_{L^{2}}^{2}+C\|\Delta d\|_{L^{2}}^{2} \leq C\left(\|\nabla u\|_{L^{2}}^{2}+\mid \Delta d \|_{L^{2}}^{2}\right)
$$

due to $1.11_{1}$, and Poincaré's inequality. With this key exponential decay-in-time rate at hand, we can obtain that both $\|\nabla u\|_{L^{2}}^{2}+\|\Delta d\|_{L^{2}}^{2}$ and $\left\|\sqrt{\rho} u_{t}\right\|_{L^{2}}^{2}+\left\|\nabla d_{t}\right\|_{L^{2}}^{2}$ decay at the same rate as $e^{-\sigma t}(\sigma>0)$ for large time (3.10) and (3.24). In fact, all these exponential decay-in-time rates are the key to obtaining the desired uniform bound (with respect to time) on the $L^{1}\left(\min \{1, T\}, T ; L^{\infty}\right)$-norm of $\nabla u$ (see $(3.37)$ ). Finally, using these a priori estimates and the fact that the velocity is divergent free, we establish the time-independent estimates on the $\|\nabla \mu(\rho)\|_{L^{q}},\|\nabla u\|_{L^{2}}^{2}$, and $\|\Delta d\|_{L^{2}}^{2}$ which guarantee the extension of local strong solutions (see Proposition 3.1).

The rest of this paper is organized as follows. In Section 2, we collect some elementary facts and inequalities that will be used later. Section 3 is devoted to the a priori estimates. Finally, we will prove Theorem 1.1 in Section 4 .

\section{Preliminaries}

In this section, we shall enumerate some auxiliary lemmas. 
We start with the following local existence of strong solutions can be shown by similar strategies as in $3,16,24]$.

Lemma 2.1. Assume that $\left(\rho_{0}, \rho_{0} u_{0}, d_{0}\right)$ satisfies 1.6$)$. Then there exists a small time $T_{0}>0$ and a unique strong solution $(\rho, u, d, P)$ to the problem $1.1-(1.4)$ in $\Omega \times\left(0, T_{0}\right)$ satisfying (1.7).

The following regularity results on the Stokes equations will be useful for our derivation of higher order a priori estimates (see [1] ).

Lemma 2.2. Assume that $\rho \in W^{1, q}, 2<q<\infty, 0 \leq \rho \leq \bar{\rho}$, and $\mu(\rho)$ satisfies (1.2) on $[0, \bar{\rho}]$. Let $(u, P) \in H_{0, \sigma}^{1} \times L^{2}$ be the unique weak solution to the following boundary value problem

$$
-\operatorname{div}(\mu(\rho) \nabla u)+\nabla P=F, \quad \operatorname{div} u=0 \quad \text { in } \Omega, \quad \int P d x=0 .
$$

Then we have the following regularity results:

- If $F \in L^{2}$, then $(u, P) \in H^{2} \times H^{1}$ and

$$
\begin{aligned}
\|u\|_{H^{2}} & \leq C\|F\|_{L^{2}}\left(1+\|\nabla \mu(\rho)\|_{L^{q}}\right)^{q /(q-2)}, \\
\|P\|_{H^{1}} & \leq C\|F\|_{L^{2}}\left(1+\|\nabla \mu(\rho)\|_{L^{q}}\right)^{(2 q-2) /(q-2)} .
\end{aligned}
$$

- If $F \in L^{r}$ for some $r \in(2, q)$, then $(u, P) \in W^{2, r} \times W^{1, r}$ and

$$
\begin{aligned}
& \|u\|_{W^{2, r}} \leq C\|F\|_{L^{r}}\left(1+\|\nabla \mu(\rho)\|_{\left.L^{q}\right)^{\frac{q r}{2(q-r)}},}\right.
\end{aligned}
$$

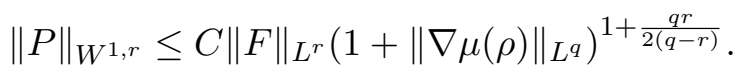

Here the constant $C$ in (2.1) and $(2.2)$ depends on $\Omega, q, r, \underline{\mu}, \bar{\mu}$.

\section{A priori estimates}

In this section, we will establish some necessary a priori bounds of local strong solutions $(\rho, u, d, P)$ to the problem (1.1)-1.4 whose existence is guaranteed by Lemma 2.1. Thus, let $T>0$ be a fixed time and $(\rho, u, d, P)$ be the smooth solution $1.1-1.4)$ on $\Omega \times(0, T]$ with smooth initial data $\left(\rho_{0}, u_{0}, d_{0}\right)$ satisfying $(1.6)$. For simplicity, we shall the letters $C$ and $C_{i}(i=1,2, \ldots)$ (except Lemma 3.6 to denote the generic constants which may be dependent on $\Omega, q, \underline{\mu}, \bar{\mu}$, and $M_{1}$, but independent of $T, M_{2}$, and $M_{3}$.

We aim to get the following key a priori estimates on $(\rho, u, d, P)$. 
Proposition 3.1. There exists some small positive constant $\epsilon_{0}$ depending only on $\Omega, q$, $\underline{\mu}, \bar{\mu}, M_{1}, M_{2}$, and $M_{3}$ such that if $(\rho, u, d, P)$ is a smooth solution of 1.1 - 1.4 on $\Omega \times(0, T]$ satisfying

$$
\begin{gathered}
\sup _{0 \leq t \leq T}\left(\bar{\rho}+\|\nabla d\|_{L^{2}}\right) \leq M_{0}, \quad \sup _{0 \leq t \leq T}\|\nabla \mu(\rho)\|_{L^{q}} \leq 4 M_{1}, \\
\sup _{0 \leq t \leq T} e^{\sigma t}\left(\underline{\mu}\|\nabla u\|_{L^{2}}^{2}+\|\Delta d\|_{L^{2}}^{2}\right) \leq 4\left[2\left(\frac{\bar{\mu}}{\mu}+1\right) M_{2}+\frac{3}{2} M_{3}\right],
\end{gathered}
$$

the following estimates hold

$$
\begin{gathered}
\sup _{0 \leq t \leq T}\left(\bar{\rho}+\|\nabla d\|_{L^{2}}\right) \leq \frac{M_{0}}{2}, \quad \sup _{0 \leq t \leq T}\|\nabla \mu(\rho)\|_{L^{q}} \leq 2 M_{1}, \\
\sup _{0 \leq t \leq T} e^{\sigma t}\left(\underline{\mu}\|\nabla u\|_{L^{2}}^{2}+\|\Delta d\|_{L^{2}}^{2}\right) \leq 2\left[2\left(\frac{\bar{\mu}}{\underline{\mu}}+1\right) M_{2}+\frac{3}{2} M_{3}\right],
\end{gathered}
$$

provided

$$
M_{0} \leq \epsilon_{0},
$$

where

$$
\begin{aligned}
& \epsilon_{0}:=\min \left\{1, \frac{1}{\sqrt{2 C_{1}}}, \frac{1}{\left(8 C_{2}\right)^{3}}, \frac{1}{\left(8 C_{2}\right)^{2} M_{3}^{1 / 2}}, \frac{1}{4 C_{3} M_{3}}, \frac{1}{\left(4 C_{3}\right)^{2} M_{3}^{9 / 2}}, \frac{1}{12 C_{4} M_{3}^{3}},\right. \\
& \frac{1}{\left[12 C_{4}\left(M_{3}^{9 / 2}+1\right)\right]^{3 / 2}}, \frac{1}{12 C_{4} M_{3}^{9 / 2}}, \frac{1}{\left(2 C_{5}\right)^{1 / 2}}, \frac{1}{2 C_{6} M_{3}^{1 / 2}}, \\
& \left(\frac{\ln 2}{3 C_{7} M_{3}\left(M_{3}^{9 / 2}+1\right)^{3 / 2}}\right)^{4 / 3},\left(\frac{\ln 2}{3 C_{7} M_{3}^{\frac{5 r-6}{2 r}} M_{2}}\right)^{r /(6 r-6)}, \\
& \left(\frac{\ln 2}{3 C_{7} M_{3}^{7 / 12}\left(M_{3}^{9 / 4}+1\right)^{7 / 6}}\right)^{4 / 3}, \frac{1}{2 C_{8}}, \frac{1}{2 C_{9}}, \frac{1}{12 C_{10} M_{3}^{\frac{5 r-6}{4 r}} M_{2}}, \\
& \left.\frac{1}{12 C_{10} M_{3}^{1 / 2}\left(M_{3}^{9 / 2}+1\right)^{3 / 4}}, \frac{1}{12 C_{10} M_{3}^{7 / 24}\left(M_{3}^{9 / 2}+1\right)^{7 / 12}}, \frac{1}{4}\right\} \text {. }
\end{aligned}
$$

Before proving Proposition 3.1, we establish some necessary a priori estimates, see Lemmas 3.23 .5 .

We begin with the nonnegativity and boundedness of density and basic energy estimates for $(\rho, u, d, P)$.

Lemma 3.2. Let $(\rho, u, d, P)$ be a smooth solution to $1.1-1.4$ on $[0, T]$. Then for

$$
\sigma:=\min \left\{\frac{1}{2 \widetilde{C} C_{p}^{2}}, \frac{\underline{\mu}}{2 C_{p}^{2} \bar{\rho}}\right\},
$$


it holds that

$$
\begin{gathered}
0 \leq \rho(x, t) \leq \sup _{x \in \Omega} \rho_{0}(x)=\bar{\rho} \\
e^{\sigma t}\left(\|\sqrt{\rho} u\|_{L^{2}}^{2}+\|\nabla d\|_{L^{2}}^{2}\right)+\int_{0}^{t} e^{\sigma t}\left(\underline{\mu}\|\nabla u\|_{L^{2}}^{2}+\|\Delta d\|_{L^{2}}^{2}\right) d t \\
\leq M_{2} \quad \text { for every } t \in[0, T]
\end{gathered}
$$

provided $\sup _{0 \leq t \leq T}\left(\bar{\rho}+\|\nabla d\|_{L^{2}}\right) \leq \epsilon_{1}:=\min \left\{1, \frac{1}{\sqrt{2 C_{1}}}\right\}$. Here, $C_{p}$ is the constant of Poincaré's inequality.

Proof. Note that (3.4) follows from the transport equation $(1.1)_{1}$ and making use of $(1.1)_{3}$ (see Lions $\left[21\right.$, Theorem 2.1]). Next, multiplying $(1.1)_{2}$ and $(1.1)_{4}$ by $u$ and $-\left(\Delta d+|\nabla d|^{2} d\right)$, respectively, summing the resulting equations up and integrating over $\Omega$, then it follows from integration by parts and using $(1.1)_{1}$ and $(1.1)_{3}$ that

$$
\frac{1}{2} \frac{d}{d t} \int\left(\rho|u|^{2}+|\nabla d|^{2}\right) d x+\int\left(\mu(\rho)|\nabla u|^{2}+\left.\left.|\Delta d+| \nabla d\right|^{2} d\right|^{2}\right) d x=0,
$$

from which, noticing that

$$
\left.\left.|\Delta d+| \nabla d\right|^{2} d\right|^{2}=|\Delta d|^{2}-|\nabla d|^{4}
$$

guaranteed by the condition $|d|=1$, one arrives at

$$
\begin{aligned}
& \frac{1}{2} \frac{d}{d t} \int\left(\rho|u|^{2}+|\nabla d|^{2}\right) d x+\int\left(\mu(\rho)|\nabla u|^{2}+|\Delta d|^{2}\right) d x \\
= & \int|\nabla d|^{4} d x \leq C\|\nabla d\|_{L^{2}}^{2}\|\nabla d\|_{H^{1}}^{2} \leq C_{1}\|\nabla d\|_{L^{2}}^{2}\|\Delta d\|_{L^{2}}^{2},
\end{aligned}
$$

which directly deduce that

$$
\frac{d}{d t} \int\left(\rho|u|^{2}+|\nabla d|^{2}\right) d x+\int\left(\underline{\mu}|\nabla u|^{2}+|\Delta d|^{2}\right) d x \leq 0
$$

provided $\sup _{0 \leq t \leq T}\|\nabla d\|_{L^{2}} \leq M_{0} \leq \epsilon_{1}:=\min \left\{1, \frac{1}{\sqrt{2 C_{1}}}\right\}$. Then, integrating 3.6 over $[0, T]$, one arrives at

$$
\|\sqrt{\rho} u\|_{L^{2}}^{2}+\|\nabla d\|_{L^{2}}^{2}+\int_{0}^{t}\left(\underline{\mu}\|\nabla u\|_{L^{2}}^{2}+\|\Delta d\|_{L^{2}}^{2}\right) d t \leq M_{2} \quad \text { for every } t \in[0, T] .
$$

Moveover, it follows from the Poincaré's inequality, and (3.4) that

$$
\begin{aligned}
\|\sqrt{\rho} u\|_{L^{2}}^{2}+\|\nabla d\|_{L^{2}}^{2} & \leq\|\rho\|_{L^{\infty}}\|u\|_{L^{2}}^{2}+\widetilde{C} C_{p}^{2}\|\Delta d\|_{L^{2}}^{2} \\
& \leq \frac{C_{p}^{2} \bar{\rho}}{\underline{\mu}}\left(\underline{\mu}\|\nabla u\|_{L^{2}}^{2}\right)+\widetilde{C} C_{p}^{2}\|\Delta d\|_{L^{2}}^{2} \\
& \leq \sigma^{-1}\left(\frac{\mu}{2}\|\nabla u\|_{L^{2}}^{2}+\frac{1}{2}\|\Delta d\|_{L^{2}}^{2}\right),
\end{aligned}
$$


where we have used the following inequality

$$
\|d\|_{H^{2}}^{2} \leq \widetilde{C}\|\Delta d\|_{L^{2}}^{2}
$$

Here, $\sigma$ is defined as in (3.3). Putting (3.7) into $(3.6)$ yields

$$
\frac{d}{d t}\left(\left(\|\sqrt{\rho} u\|_{L^{2}}^{2}+\|\nabla d\|_{L^{2}}^{2}\right)+\sigma\left(\|\sqrt{\rho} u\|_{L^{2}}^{2}+\|\nabla d\|_{L^{2}}^{2}\right)\right)+\frac{1}{2}\left(\underline{\mu}\|\nabla u\|_{L^{2}}^{2}+\|\Delta d\|_{L^{2}}^{2}\right) \leq 0,
$$

that is

$$
\frac{d}{d t}\left(e^{\sigma t}\left(\|\sqrt{\rho} u\|_{L^{2}}^{2}+\|\nabla d\|_{L^{2}}^{2}\right)\right)+e^{\sigma t}\left(\underline{\mu}\|\nabla u\|_{L^{2}}^{2}+\|\Delta d\|_{L^{2}}^{2}\right) \leq 0,
$$

which, integrated over $[0, t]$ gives $(3.5)$. The proof of Lemma 3.2 is completed.

Lemma 3.3. Let $(\rho, u, d, P)$ be a smooth solution to (1.1) (1.4) satisfying (3.1). Then there exists a generic positive constant $C$ depending only on $\Omega, q, \mu, \bar{\mu}, M_{1}, M_{2}$, and $M_{3}$ such that

$$
\begin{aligned}
& \sup _{0 \leq t \leq \zeta(T)}\left(\underline{\mu}\|\nabla u\|_{L^{2}}^{2}+\|\Delta d\|_{L^{2}}^{2}\right)+\int_{0}^{\zeta(T)}\left(\left\|\sqrt{\rho} u_{t}\right\|_{L^{2}}^{2}+\left\|\nabla d_{t}\right\|_{L^{2}}^{2}\right) d t \leq M_{3}, \\
& \sup _{\zeta(T) \leq t \leq T} e^{\sigma t}\left(\underline{\mu}\|\nabla u\|_{L^{2}}^{2}+\|\Delta d\|_{L^{2}}^{2}\right)+\int_{\zeta(T)}^{T} e^{\sigma t}\left(\left\|\sqrt{\rho} u_{t}\right\|_{L^{2}}^{2}+\left\|\nabla d_{t}\right\|_{L^{2}}^{2}\right) d t \leq C M_{3},
\end{aligned}
$$

provided $\sup _{0 \leq t \leq T}\left(\bar{\rho}+\|\nabla d\|_{L^{2}}\right) \leq \epsilon_{2}:=\min \left\{\epsilon_{1}, \frac{1}{\left(8 C_{2}\right)^{3}}, \frac{1}{\left(8 C_{2}\right)^{2} M_{3}^{1 / 2}}, \frac{1}{4 C_{3} M_{3}}, \frac{1}{\left(4 C_{3}\right)^{2} M_{3}^{9 / 2}}\right.$, $\left.\frac{1}{\left[12 C_{4}\left(M_{3}^{9 / 2}+1\right)\right]^{3 / 2}}, \frac{1}{12 C_{4} M_{3}^{3}}, \frac{1}{12 C_{4} M_{3}^{9 / 2}}, \frac{1}{\left(2 C_{5}\right)^{1 / 2}}\right\}$. Here, $\zeta(t)$ is defined by

$$
\zeta(t):=\min \{1, t\}
$$

Proof. First, by $(1.1)_{1}$, we obtain the following equation

$$
\mu(\rho)_{t}+u \cdot \nabla \mu(\rho)=0
$$

Next, multiplying 1.1$)_{2}$ by $u_{t}$ in $L^{2}$, we obtain after using integration by parts over $\Omega$ and $(1.1)_{3}$ that

$$
\begin{aligned}
& \frac{1}{2} \frac{d}{d t} \int \mu(\rho)|\nabla u|^{2} d x+\int \rho\left|u_{t}\right|^{2} d x \\
= & -\int \rho u \cdot \nabla u \cdot u_{t} d x-\frac{1}{2} \int u \cdot \nabla \mu(\rho)|\nabla u|^{2} d x+\int \nabla d \odot \nabla d: \nabla u_{t} d x \\
= & \frac{d}{d t} \int \nabla d \odot \nabla d: \nabla u d x-\int(\nabla d \odot \nabla d)_{t}: \nabla u d x-\int \rho u \cdot \nabla u \cdot u_{t} d x \\
& -\frac{1}{2} \int u \cdot \nabla \mu(\rho)|\nabla u|^{2} d x \\
= & : \frac{d}{d t} \int \nabla d \odot \nabla d: \nabla u d x+\sum_{i=1}^{3} \mathcal{I}_{1 i} .
\end{aligned}
$$


Now, we will use the Gagliardo-Nirenberg inequality, Poincaré's inequality, (3.1), (3.5), and (3.4) to estimate each term on the right hand of $(3.11)$ as follows:

$$
\begin{aligned}
\mathcal{I}_{11} & \leq C\left\|\nabla d_{t}\right\|_{L^{2}}\|\nabla d\|_{L^{4}}\|\nabla u\|_{L^{4}} \leq C\left\|\nabla d_{t}\right\|_{L^{2}}\|\nabla d\|_{L^{2}}^{1 / 2}\left\|\nabla^{2} d\right\|_{L^{2}}^{1 / 2}\|\nabla u\|_{H^{1}} \\
& \leq \frac{1}{8}\left\|\nabla d_{t}\right\|_{L^{2}}^{2}+C\|\nabla d\|_{L^{2}}\|\Delta d\|_{L^{2}}\|\nabla u\|_{H^{1}}^{2} \leq \frac{1}{8}\left\|\nabla d_{t}\right\|_{L^{2}}^{2}+C M_{3}\|\nabla u\|_{H^{1}}^{2}, \\
\mathcal{I}_{12} & \leq C \bar{\rho}^{1 / 2}\left\|\sqrt{\rho} u_{t}\right\|_{L^{2}}\|u\|_{L^{\infty}}\|\nabla u\|_{L^{2}} \leq \frac{1}{4}\left\|\sqrt{\rho} u_{t}\right\|_{L^{2}}^{2}+C\left\|\nabla^{2} u\right\|_{L^{2}}\|u\|_{L^{2}}\|\nabla u\|_{L^{2}}^{2} \\
& \leq \frac{1}{4}\left\|\sqrt{\rho} u_{t}\right\|_{L^{2}}^{2}+C\|\nabla u\|_{L^{2}}^{2}\|\nabla u\|_{H^{1}}^{2} \leq \frac{1}{4}\left\|\sqrt{\rho} u_{t}\right\|_{L^{2}}^{2}+C M_{3}\|\nabla u\|_{H^{1}}^{2}, \\
\mathcal{I}_{13} & \leq C\|\nabla \mu(\rho)\|_{L^{q}}\|u\|_{L^{2 q /(q-2)}}\|\nabla u\|_{L^{4}}^{2} \leq C\|\nabla u\|_{L^{2}}\|\nabla u\|_{H^{1}}^{2} \leq C M_{3}^{1 / 2}\|\nabla u\|_{H^{1}}^{2},
\end{aligned}
$$

where we have used the following fact that

$$
\left\|\sqrt{\rho_{0}} u_{0}\right\|_{L^{2}}^{2}+\left\|\nabla d_{0}\right\|_{L^{2}}^{2} \leq\left\|\rho_{0}\right\|_{L^{\infty}}\left\|u_{0}\right\|_{L^{2}}^{2}+C\left\|\Delta d_{0}\right\|_{L^{2}}^{2} \leq C\left(\left\|\nabla u_{0}\right\|_{L^{2}}^{2}+\left\|\Delta d_{0}\right\|_{L^{2}}^{2}\right) .
$$

Substituting (3.12)-(3.14) into (3.11), using (3.1), yields that

$$
\begin{aligned}
& \frac{1}{2} \frac{d}{d t} \int \mu(\rho)|\nabla u|^{2} d x+\int \rho\left|u_{t}\right|^{2} d x \\
\leq & \frac{d}{d t} \int \nabla d \odot \nabla d: \nabla u d x+\frac{1}{8}\left\|\nabla d_{t}\right\|_{L^{2}}^{2}+\frac{1}{4}\left\|\sqrt{\rho} u_{t}\right\|_{L^{2}}^{2}+C\left(M_{3}+M_{3}^{1 / 2}\right)\|\nabla u\|_{H^{1}}^{2} .
\end{aligned}
$$

Next, multiplying (1.1) 4 by $\Delta d_{t}$ and integrating over $\Omega$, using (3.15), (3.1), GagliardoNirenberg, Poincaré's, and Young's inequalities, we obtain, bearing in mind the facts that $|d|=1, u=0$ and $\nabla d_{0}^{\prime}=0$ on $\partial \Omega$ that

$$
\begin{aligned}
& \frac{1}{2} \frac{d}{d t} \int|\Delta d|^{2} d x+\int\left|\nabla d_{t}\right|^{2} d x \\
= & \int u \cdot \nabla d \cdot \Delta d_{t} d x-\int|\nabla d|^{2} d \cdot \Delta d_{t} d x \\
\leq & \frac{1}{8}\left\|\nabla d_{t}\right\|_{L^{2}}^{2}+C \int\left(|\nabla(u \cdot \nabla d)|^{2}+\left|\nabla\left(|\nabla d|^{2} d\right)\right|^{2}\right) d x \\
\leq & \frac{1}{8}\left\|\nabla d_{t}\right\|_{L^{2}}^{2}+C\left(\left\|\left|\nabla u\left\|\nabla d\left|\left\|_{L^{2}}^{2}+\right\|\right|\right\| \nabla^{2} d\right|\right\|_{L^{2}}^{2}+\|\nabla d\|_{L^{6}}^{6}+\left\|\left|\nabla d\left\|\nabla^{2} d \mid\right\|_{L^{2}}^{2}\right)\right.\right. \\
\leq & \frac{1}{8}\left\|\nabla d_{t}\right\|_{L^{2}}^{2}+C\|\nabla u\|_{L^{2}}^{2}\|\nabla d\|_{L^{\infty}}^{2}+C\|u\|_{L^{4}}^{2}\left\|\nabla^{2} d\right\|_{L^{4}}^{2} \\
& +C\|\nabla d\|_{L^{\infty}}^{4}\|\nabla d\|_{L^{2}}^{2}+C\|\nabla d\|_{L^{4}}^{2}\left\|\nabla^{2} d\right\|_{L^{4}}^{2} \\
\leq & \frac{1}{8}\left\|\nabla d_{t}\right\|_{L^{2}}^{2}+C\|\nabla d\|_{L^{2}}\left\|\nabla^{3} d\right\|_{L^{2}}\|\nabla u\|_{L^{2}}^{2}+C\|\nabla u\|_{L^{2}}^{2}\left\|\nabla^{3} d\right\|_{L^{2}}^{3 / 2}\|\nabla d\|_{L^{2}}^{1 / 2} \\
& +C\left\|\nabla^{3} d\right\|_{L^{2}}^{2}\|\nabla d\|_{L^{2}}^{4}+C\|\nabla d\|_{L^{2}}\left\|\nabla^{2} d\right\|_{L^{2}}^{2}\left\|\nabla^{3} d\right\|_{L^{2}} \\
\leq & \frac{1}{8}\left\|\nabla d_{t}\right\|_{L^{2}}^{2}+C\left(\|\nabla d\|_{L^{2}}^{2}+\|\nabla d\|_{L^{2}}^{2 / 3}+\|\nabla d\|_{L^{2}}^{4}+M_{3}^{1 / 2}\|\nabla d\|_{L^{2}}\right)\left\|\nabla^{3} d\right\|_{L^{2}}^{2} \\
& +C\|\nabla u\|_{L^{2}}^{4}+C\|\nabla u\|_{L^{2}}^{8}
\end{aligned}
$$




$$
\begin{aligned}
\leq & \frac{1}{8}\left\|\nabla d_{t}\right\|_{L^{2}}^{2}+C\left(\|\nabla d\|_{L^{2}}^{2}+\|\nabla d\|_{L^{2}}^{2 / 3}+\|\nabla d\|_{L^{2}}^{4}+M_{3}^{1 / 2}\|\nabla d\|_{L^{2}}\right)\left\|\nabla^{3} d\right\|_{L^{2}}^{2} \\
& +C\left(M_{3}+M_{3}^{3}\right)\|\nabla u\|_{H^{1}}^{2} .
\end{aligned}
$$

Adding (3.16) to (3.17), it holds that

$$
\begin{aligned}
& \frac{1}{2} \frac{d}{d t} \int\left(\mu(\rho)|\nabla u|^{2}+|\Delta d|^{2}\right) d x+\left\|\sqrt{\rho} u_{t}\right\|_{L^{2}}^{2}+\left\|\nabla d_{t}\right\|_{L^{2}}^{2} \\
\leq & \frac{d}{d t} \int \nabla d \odot \nabla d: \nabla u d x+\frac{1}{4}\left(\left\|\nabla d_{t}\right\|_{L^{2}}^{2}+\left\|\sqrt{\rho} u_{t}\right\|_{L^{2}}^{2}\right)+C M_{3}^{3}\|\nabla u\|_{H^{1}}^{2} \\
& +C\left(\|\nabla d\|_{L^{2}}^{2}+\|\nabla d\|_{L^{2}}^{2 / 3}+\|\nabla d\|_{L^{2}}^{4}+M_{3}^{1 / 2}\|\nabla d\|_{L^{2}}\right)\left\|\nabla^{3} d\right\|_{L^{2}}^{2} .
\end{aligned}
$$

Moreover, we estimate $\|\nabla u\|_{H^{1}}$ and $\left\|\nabla^{3} d\right\|_{L^{2}}$. According to Lemma 2.2 with $F=$ $-\rho u_{t}-\rho u \cdot \nabla u-\operatorname{div}(\nabla d \odot \nabla d)$, using (3.4), (3.15), and (3.1), we derive

$$
\begin{aligned}
& \|\nabla u\|_{H^{1}}+\|P\|_{H^{1}} \\
\leq & C\left[\left(1+\|\nabla \mu(\rho)\|_{L^{q}}\right)^{q /(q-2)}+\left(1+\|\nabla \mu(\rho)\|_{\left.\left.L^{q}\right)^{(2 q-2) /(q-2)}\right]\|F\|_{L^{2}}}\right.\right. \\
\leq & C\left(\bar{\rho}\|u\|_{L^{\infty}}\|\nabla u\|_{L^{2}}+\bar{\rho}^{1 / 2}\left\|\sqrt{\rho} u_{t}\right\|_{L^{2}}+\|\nabla d\|_{L^{\infty}}\left\|\nabla^{2} d\right\|_{L^{2}}\right) \\
\leq & C \bar{\rho}\left\|\nabla^{2} u\right\|_{L^{2}}^{1 / 2}\|u\|_{L^{2}}^{1 / 2}\|\nabla u\|_{L^{2}}^{2}+C \bar{\rho}^{1 / 2}\left\|\sqrt{\rho} u_{t}\right\|_{L^{2}}+C\|\nabla d\|_{L^{2}}^{1 / 2}\left\|\nabla^{3} d\right\|_{L^{2}}^{1 / 2}\left\|\nabla^{2} d\right\|_{L^{2}}^{2} \\
\leq & C \bar{\rho} M_{3}\|\nabla u\|_{H^{1}}+C \bar{\rho}^{1 / 2}\left\|\sqrt{\rho} u_{t}\right\|_{L^{2}}+C\|\nabla d\|_{L^{2}}^{1 / 2}\left\|\nabla^{3} d\right\|_{L^{2}}\|\Delta d\|_{L^{2}}^{3 / 2} \\
\leq & C \bar{\rho} M_{3}\|\nabla u\|_{H^{1}}+C \bar{\rho}^{1 / 2}\left\|\sqrt{\rho} u_{t}\right\|_{L^{2}}+C M_{3}^{3 / 4}\|\nabla d\|_{L^{2}}^{1 / 2}\left\|\nabla^{3} d\right\|_{L^{2}} .
\end{aligned}
$$

Using $H^{k}$ estimate of the elliptic systems, it is easy to deduce from 1.1$)_{4}$ that

$$
\begin{aligned}
\left\|\nabla^{3} d\right\|_{L^{2}} \leq & C\left\|\nabla d_{t}+\nabla(u \cdot \nabla d)-\nabla\left(|\nabla d|^{2} d\right)\right\|_{L^{2}} \\
\leq & C_{2}\left(\|\nabla d\|_{L^{2}}+\|\nabla d\|_{L^{2}}^{1 / 3}+\|\nabla d\|_{L^{2}}^{2}+M_{3}^{1 / 4}\|\nabla d\|_{L^{2}}^{1 / 2}\right)\left\|\nabla^{3} d\right\|_{L^{2}} \\
& +C\left\|\nabla d_{t}\right\|_{L^{2}}+C M_{3}^{3 / 2}\|\nabla u\|_{H^{1}}
\end{aligned}
$$

which directly leads to

$$
\left\|\nabla^{3} d\right\|_{L^{2}} \leq C M_{3}^{3 / 2}\|\nabla u\|_{H^{1}}+C\left\|\nabla d_{t}\right\|_{L^{2}}
$$

provided $\sup _{0 \leq t \leq T}\|\nabla d\|_{L^{2}} \leq M_{0} \leq \epsilon_{2}:=\min \left\{\epsilon_{1}, \frac{1}{\left(8 C_{2}\right)^{3}}, \frac{1}{\left(8 C_{2}\right)^{2} M_{3}^{1 / 2}}\right\}$. Inserting 3.19 into 3.18, we have

$$
\|\nabla u\|_{H^{1}} \leq C_{3}\left(\bar{\rho} M_{3}+\|\nabla d\|_{L^{2}}^{1 / 2} M_{3}^{9 / 4}\right)\|\nabla u\|_{H^{1}}+\bar{\rho}^{1 / 2}\left\|\sqrt{\rho} u_{t}\right\|_{L^{2}}+C M_{3}^{3 / 4}\|\nabla d\|_{L^{2}}^{1 / 2}\left\|\nabla d_{t}\right\|_{L^{2}},
$$

that is

$$
\|\nabla u\|_{H^{1}} \leq C\left(\bar{\rho}^{1 / 2}+M_{3}^{3 / 4}\|\nabla d\|_{L^{2}}^{1 / 2}\right)\left(\left\|\sqrt{\rho} u_{t}\right\|_{L^{2}}+\left\|\nabla d_{t}\right\|_{L^{2}}\right),
$$


provided $\sup _{0 \leq t \leq T}\left(\bar{\rho}+\|\nabla d\|_{L^{2}}\right) \leq M_{0} \leq \epsilon_{3}:=\min \left\{\epsilon_{2}, \frac{1}{4 C_{3} M_{3}}, \frac{1}{\left(4 C_{3}\right)^{2} M_{3}^{9 / 2}}\right\}$. Combining (3.19) and (3.20), yields that

$$
\begin{aligned}
\left\|\nabla^{3} d\right\|_{L^{2}} & \leq C M_{3}^{3 / 2}\left(\bar{\rho}^{1 / 2}+M_{3}^{3 / 4}\|\nabla d\|_{L^{2}}^{1 / 2}\right)\left(\left\|\sqrt{\rho} u_{t}\right\|_{L^{2}}+\left\|\nabla d_{t}\right\|_{L^{2}}\right)+C\left\|\nabla d_{t}\right\|_{L^{2}} \\
& \leq C\left(M_{3}^{9 / 4}+1\right)\left(\left\|\sqrt{\rho} u_{t}\right\|_{L^{2}}+\left\|\nabla d_{t}\right\|_{L^{2}}\right) .
\end{aligned}
$$

Thus, it follows from 3.20 and 3.21 that

$$
\begin{aligned}
& \frac{1}{2} \frac{d}{d t} \int\left(\mu(\rho)|\nabla u|^{2}+|\Delta d|^{2}\right) d x+\left\|\sqrt{\rho} u_{t}\right\|_{L^{2}}^{2}+\left\|\nabla d_{t}\right\|_{L^{2}}^{2} \\
\leq & \frac{d}{d t} \int \nabla d \odot \nabla d: \nabla u d x+\frac{1}{4}\left(\left\|\nabla d_{t}\right\|_{L^{2}}^{2}+\left\|\sqrt{\rho} u_{t}\right\|_{L^{2}}^{2}\right) \\
& +C\left[\bar{\rho} M_{3}^{3}+M_{3}^{9 / 2}\|\nabla d\|_{L^{2}}+\left(M_{3}^{9 / 2}+1\right)\left(\|\nabla d\|_{L^{2}}^{2}+\|\nabla d\|_{L^{2}}^{2 / 3}+\|\nabla d\|_{L^{2}}^{4}\right)\right] \\
& \times\left(\left\|\sqrt{\rho} u_{t}\right\|_{L^{2}}^{2}+\left\|\nabla d_{t}\right\|_{L^{2}}^{2}\right) \\
\leq & \frac{d}{d t} \int \nabla d \odot \nabla d: \nabla u d x+\frac{1}{4}\left(\left\|\nabla d_{t}\right\|_{L^{2}}^{2}+\left\|\sqrt{\rho} u_{t}\right\|_{L^{2}}^{2}\right) \\
& +C_{4}\left(\bar{\rho} M_{3}^{3}+M_{3}^{9 / 2}\|\nabla d\|_{L^{2}}+\left(M_{3}^{9 / 2}+1\right)\|\nabla d\|_{L^{2}}^{2 / 3}\right)\left(\left\|\sqrt{\rho} u_{t}\right\|_{L^{2}}^{2}+\left\|\nabla d_{t}\right\|_{L^{2}}^{2}\right),
\end{aligned}
$$

which, yields that

$$
\frac{1}{2} \frac{d}{d t} \int\left(\mu(\rho)|\nabla u|^{2}+|\Delta d|^{2}\right) d x+\left\|\sqrt{\rho} u_{t}\right\|_{L^{2}}^{2}+\left\|\nabla d_{t}\right\|_{L^{2}}^{2} \leq 2 \frac{d}{d t} \int \nabla d \odot \nabla d: \nabla u d x
$$

provided $\sup _{0 \leq t \leq T}\left(\bar{\rho}+\|\nabla d\|_{L^{2}}\right) \leq M_{0} \leq \epsilon_{4}:=\min \left\{\epsilon_{3}, \frac{1}{12 C_{4} M_{3}^{3}}, \frac{1}{\left[12 C_{4}\left(M_{3}^{9 / 2}+1\right)\right]^{3 / 2}}, \frac{1}{12 C_{4} M_{3}^{9 / 2}}\right\}$. Integrating 3.22 over $[0, \zeta(T)]$, from 1.2 and $(3.8)$, gives

$$
\begin{aligned}
& \sup _{0 \leq t \leq \zeta(T)}\left(\underline{\mu}\|\nabla u\|_{L^{2}}^{2}+\|\Delta d\|_{L^{2}}^{2}\right)+\int_{0}^{\zeta(T)}\left(\left\|\sqrt{\rho} u_{t}\right\|_{L^{2}}^{2}+\left\|\nabla d_{t}\right\|_{L^{2}}^{2}\right) d t \\
& \leq \frac{\mu}{\overline{2}} \sup _{0 \leq t \leq \zeta(T)}\|\nabla u\|_{L^{2}}^{2}+C_{5} \sup _{0 \leq t \leq \zeta(T)}\left[\|\nabla d\|_{L^{2}}^{2}\|\Delta d\|_{L^{2}}^{2}\right]+M_{3} .
\end{aligned}
$$

From the above inequality, we get

$$
\sup _{0 \leq t \leq \zeta(T)}\left(\underline{\mu}\|\nabla u\|_{L^{2}}^{2}+\|\Delta d\|_{L^{2}}^{2}\right)+\int_{0}^{\zeta(T)}\left(\left\|\sqrt{\rho} u_{t}\right\|_{L^{2}}^{2}+\left\|\nabla d_{t}\right\|_{L^{2}}^{2}\right) d t \leq M_{3},
$$

provided $\sup _{0 \leq t \leq T}\|\nabla d\|_{L^{2}} \leq M_{0} \leq \epsilon_{5}:=\min \left\{\epsilon_{4}, \frac{1}{\left(2 C_{5}\right)^{1 / 2}}\right\}$.

Finally, it remains to estimate 3.10 . In fact, multiplying 3.22 by $e^{\sigma t}$ and integrating the result inequality over $[\zeta(T), T]$, we obtain

$$
\begin{gathered}
\sup _{\zeta(T) \leq t \leq T} e^{\sigma t}\left(\underline{\mu}\|\nabla u\|_{L^{2}}^{2}+\|\Delta d\|_{L^{2}}^{2}\right)+\int_{\zeta(T)}^{T} e^{\sigma t}\left(\left\|\sqrt{\rho} u_{t}\right\|_{L^{2}}^{2}+\left\|\nabla d_{t}\right\|_{L^{2}}^{2}\right) d t \\
\leq C \sup _{\zeta(T) \leq t \leq T} e^{\sigma t}\left[\|\nabla d\|_{L^{2}}^{2}\|\Delta d\|_{L^{2}}^{2}\right]+\int_{\zeta(T)}^{T} e^{\sigma t}\left(\bar{\mu}\|\nabla u\|_{L^{2}}^{2}+\|\Delta d\|_{L^{2}}^{2}\right) d t
\end{gathered}
$$




$$
\begin{aligned}
& +\sup _{\zeta(T) \leq t \leq T} \frac{\mu}{\overline{2}}\left[e^{\sigma t}\|\nabla u\|_{L^{2}}^{2}\right]+C \int_{\zeta(T)}^{T} e^{\sigma t}\|\nabla d\|_{L^{4}}^{2}\|\nabla u\|_{L^{2}} d t+e^{\sigma \zeta(T)} M_{3} \\
\leq & \left.C_{5} \sup _{\zeta(T) \leq t \leq T} e^{\sigma t}\left[\|\nabla d\|_{L^{2}}^{2}\|\Delta d\|_{L^{2}}^{2}\right)\right]+\int_{\zeta(T)}^{T} e^{\sigma t}\left(\bar{\mu}\|\nabla u\|_{L^{2}}^{2}+\|\Delta d\|_{L^{2}}^{2}\right) d t+e^{\sigma \zeta(T)} M_{3} \\
& +\sup _{\zeta(T) \leq t \leq T} \frac{\mu}{\frac{\mu}{2}}\left[e^{\sigma t}\|\nabla u\|_{L^{2}}^{2}\right]+C \sup _{\zeta(T) \leq t \leq T}\|\nabla d\|_{L^{2}} \int_{\zeta(T)}^{T} e^{\sigma t}\left(\underline{\mu}\|\nabla u\|_{L^{2}}^{2}+\|\Delta d\|_{L^{2}}^{2}\right) d t
\end{aligned}
$$

which directly deduce from $(3.8),(3.15)$, and (3.5) that

$$
\sup _{\zeta(T) \leq t \leq T} e^{\sigma t}\left(\underline{\mu}\|\nabla u\|_{L^{2}}^{2}+\|\Delta d\|_{L^{2}}^{2}\right)+\int_{\zeta(T)}^{T} e^{\sigma t}\left(\left\|\sqrt{\rho} u_{t}\right\|_{L^{2}}^{2}+\left\|\nabla d_{t}\right\|_{L^{2}}^{2}\right) d t \leq C M_{3},
$$

provided $\sup _{0 \leq t \leq T}\|\nabla d\|_{L^{2}} \leq M_{0} \leq \epsilon_{5}$. The proof of Lemma 3.3 is finished.

Lemma 3.4. Let $(\rho, u, d, P)$ be a smooth solution to (1.1)-1.4 satisfying (3.1). Then there exists a generic positive constant $C$ depending only on $\Omega, q, \mu, \bar{\mu}, M_{1}, M_{2}$, and $M_{3}$ such that

$$
\begin{gathered}
\sup _{0 \leq t \leq \zeta(T)}\left[t\left(\left\|\sqrt{\rho} u_{t}\right\|_{L^{2}}^{2}+\left\|\nabla d_{t}\right\|_{L^{2}}^{2}\right)\right]+\int_{0}^{\zeta(T)} t\left(\underline{\mu}\left\|\nabla u_{t}\right\|_{L^{2}}^{2}+\left\|\Delta d_{t}\right\|_{L^{2}}^{2}\right) d t \leq 2 M_{3}, \\
\sup _{\zeta(T) \leq t \leq T}\left[e^{\sigma t}\left(\left\|\sqrt{\rho} u_{t}\right\|_{L^{2}}^{2}+\left\|\nabla d_{t}\right\|_{L^{2}}^{2}\right)\right]+\int_{\zeta(T)}^{T} e^{\sigma t}\left(\underline{\mu}\left\|\nabla u_{t}\right\|_{L^{2}}^{2}+\left\|\Delta d_{t}\right\|_{L^{2}}^{2}\right) d t \\
\leq C M_{3}^{2}\left(M_{3}^{9 / 2}+1\right)^{3}, \\
\sup _{\zeta(T) \leq t \leq T} e^{\sigma t}\left(\|\nabla u\|_{H^{1}}^{2}+\left\|\nabla^{3} d\right\|_{L^{2}}^{2}\right) \leq C M_{3}^{2}\left(M_{3}^{9 / 2}+1\right)^{4},
\end{gathered}
$$

provided $\sup _{0 \leq t \leq T}\|\nabla d\|_{L^{2}} \leq M_{0} \leq \epsilon_{6}:=\min \left\{\epsilon_{5}, \frac{1}{2 C_{6} M_{3}^{1 / 2}}\right\}$.

Proof. Differentiating 1.1$]_{2}$ with respect to time variable $t$ gives

(3.26) $\rho u_{t t}+\rho u \cdot \nabla u_{t}-\operatorname{div}(\mu(\rho) \nabla u)_{t}=-\rho_{t}\left(u_{t}+u \cdot \nabla u\right)-\rho u_{t} \cdot \nabla u-\nabla P_{t}-\operatorname{div}(\nabla d \odot \nabla d)_{t}$.

Multiplying (3.26) by $u_{t}$ and integrating the resulting equality by parts over $\Omega$, we obtain after applying $(1.1)_{1}$ and the divergence free condition $(1.1)_{3}$ that

$$
\begin{aligned}
& \frac{1}{2} \frac{d}{d t} \int \rho\left|u_{t}\right|^{2} d x+\int \mu(\rho)\left|\nabla u_{t}\right|^{2} d x \\
= & \int\left[(\nabla d \odot \nabla d)_{t}: \nabla u_{t}-\rho\left(u_{t} \cdot \nabla\right) u \cdot u_{t}+\operatorname{div}(\rho u)\left(u_{t}+u \cdot \nabla u\right) \cdot u_{t}-\mu(\rho)_{t} \nabla u \cdot \nabla u_{t}\right] d x \\
= & \int\left\{(\nabla d \odot \nabla d)_{t}: \nabla u_{t}-\rho\left(u_{t} \cdot \nabla\right) u \cdot u_{t}-\rho u \cdot \nabla\left(\left|u_{t}\right|^{2}\right)+\rho u \cdot \nabla\left(u \cdot \nabla u \cdot u_{t}\right)\right. \\
& \left.\quad-\mu(\rho)_{t} \nabla u \cdot \nabla u_{t}\right\} d x
\end{aligned}
$$




$$
\begin{aligned}
\leq & C \int \rho|u|\left|u_{t}\right|\left(|\nabla u|^{2}+|u|\left|\nabla^{2} u\right|\right) d x+C \int \rho|u|^{2}|\nabla u|\left|\nabla u_{t}\right| d x+C \int \rho|u|\left|u_{t}\right|\left|\nabla u_{t}\right| d x \\
& +C \int \rho\left|u_{t}\right|^{2}|\nabla u| d x+C \int|\nabla d|\left|\nabla d_{t}\right|\left|\nabla u_{t}\right| d x+C \int|u||\nabla \mu(\rho)||\nabla u|\left|\nabla u_{t}\right| d x \\
= & \sum_{i=1}^{6} \mathcal{I}_{2 i} .
\end{aligned}
$$

Now, we use (3.4), 3.20, 3.21, the Gagliardo-Nirenberg, Poincaré's and Hölder's inequalities to estimate each term on the right-hand side of (3.27) as follows:

$$
\begin{aligned}
\mathcal{I}_{21} & \leq C \bar{\rho}\left\|u_{t}\right\|_{L^{4}}\|u\|_{L^{4}}\|\nabla u\|_{L^{4}}^{2}+C \bar{\rho}\left\|u_{t}\right\|_{L^{2}}\|u\|_{L^{\infty}}^{2}\left\|\nabla^{2} u\right\|_{L^{2}} \\
& \leq C \bar{\rho}\left\|\nabla u_{t}\right\|_{L^{2}}\|\nabla u\|_{L^{2}}\|\nabla u\|_{H^{1}}^{2} \leq \delta \underline{\mu}\left\|\nabla u_{t}\right\|_{L^{2}}^{2}+C \bar{\rho}^{2}\|\nabla u\|_{L^{2}}^{2}\|\nabla u\|_{H^{1}}^{4}, \\
\mathcal{I}_{22} & \leq C \bar{\rho}\|u\|_{L^{\infty}}^{2}\|\nabla u\|_{L^{2}}\left\|\nabla u_{t}\right\|_{L^{2}} \leq C \bar{\rho}\|u\|_{L^{2}}\|\nabla u\|_{H^{1}}\|\nabla u\|_{L^{2}}\left\|\nabla u_{t}\right\|_{L^{2}} \\
& \leq \delta \underline{\mu}\left\|\nabla u_{t}\right\|_{L^{2}}^{2}+C \bar{\rho}^{2}\|\nabla u\|_{L^{2}}^{2}\|\nabla u\|_{H^{1}}^{4}, \\
\mathcal{I}_{23} & \leq C \bar{\rho} 1 / 2\|u\|_{L^{\infty}}\left\|\nabla u_{t}\right\|_{L^{2}}\left\|\sqrt{\rho} u_{t}\right\|_{L^{2}} \leq C \bar{\rho}^{1 / 2}\|u\|_{L^{2}}^{1 / 2}\|\nabla u\|_{H^{1}}^{1 / 2}\left\|\nabla u_{t}\right\|_{L^{2}}\left\|\sqrt{\rho} u_{t}\right\|_{L^{2}} \\
& \leq \delta \underline{\mu}\left\|\nabla u_{t}\right\|_{L^{2}}^{2}+C \bar{\rho}\|\nabla u\|_{H^{1}}^{2}\left\|\sqrt{\rho} u_{t}\right\|_{L^{2}}^{2}, \\
\mathcal{I}_{24} & \leq C \bar{\rho} 1 / 2\left\|\sqrt{\rho} u_{t}\right\|_{L^{2}}\left\|u_{t}\right\|_{L^{4}}\|\nabla u\|_{L^{4}} \leq C \bar{\rho}^{1 / 2}\left\|\nabla u_{t}\right\|_{L^{2}}\|\nabla u\|_{H^{1}}\left\|\sqrt{\rho} u_{t}\right\|_{L^{2}} \\
& \leq \delta \underline{\mu}\left\|\nabla u_{t}\right\|_{L^{2}}^{2}+C \bar{\rho}\|\nabla u\|_{H^{1}}^{2}\left\|\sqrt{\rho} u_{t}\right\|_{L^{2}}^{2}, \\
\mathcal{I}_{25} & \leq C\|\nabla d\|_{L^{4}}\left\|\nabla d_{t}\right\|_{L^{4}}\left\|\nabla u_{t}\right\|_{L^{2}} \leq C\|\nabla d\|_{L^{2}}^{1 / 2}\|\Delta d\|_{L^{2}}^{1 / 2}\left\|\Delta d_{t}\right\|_{L^{2}}\left\|\nabla u_{t}\right\|_{L^{2}} \\
& \leq \delta \underline{\mu}\left\|\nabla u_{t}\right\|_{L^{2}}^{2}+C M_{3}^{1 / 2}\|\nabla d\|_{L^{2}}\left\|\Delta d_{t}\right\|_{L^{2}}^{2}, \\
\mathcal{I}_{26} & \leq C\|u\|_{L^{\infty}}\|\nabla \mu(\rho)\|\left\|_{L^{q}}\right\| \nabla u\left\|_{L^{2 q} /(q-2)}\right\| \nabla u_{t} \|_{L^{2}} \\
& \leq C\|u\|_{L^{2}}^{1 / 2}\|\nabla u\|_{H^{1}}^{1 / 2}\|\nabla \mu(\rho)\|_{L^{q}}\|\nabla u\|_{H^{1}}\left\|\nabla u_{t}\right\|_{L^{2}} \leq \delta \underline{\mu}\left\|\nabla u_{t}\right\|_{L^{2}}^{2}+C\|\nabla u\|_{H^{1}}^{2}\|\nabla u\|_{H^{1}}^{2} .
\end{aligned}
$$

Substituting the estimates of $\mathcal{I}_{2 i}(i=1,2, \ldots, 6)$ into (3.27), and then using (3.1), we obtain

$$
\begin{aligned}
& \frac{1}{2} \frac{d}{d t} \int \rho\left|u_{t}\right|^{2} d x+\int \mu(\rho)\left|\nabla u_{t}\right|^{2} d x \\
\leq & \delta \underline{\mu}\left\|\nabla u_{t}\right\|_{L^{2}}^{2}+C\left(M_{3}+1\right)\|\nabla u\|_{H^{1}}^{2}\left(\left\|\sqrt{\rho} u_{t}\right\|_{L^{2}}^{2}+\|\nabla u\|_{H^{1}}^{2}\right) \\
& +C M_{3}^{1 / 2}\|\nabla d\|_{L^{2}}\left\|\Delta d_{t}\right\|_{L^{2}}^{2} \\
\leq & \delta \underline{\mu}\left\|\nabla u_{t}\right\|_{L^{2}}^{2}+C\left(M_{3}^{3 / 2}+1\right)^{2}\|\nabla u\|_{H^{1}}^{2}\left(\left\|\sqrt{\rho} u_{t}\right\|_{L^{2}}^{2}+\left\|\nabla d_{t}\right\|_{L^{2}}^{2}\right) \\
& +C M_{3}^{1 / 2}\|\nabla d\|_{L^{2}}\left\|\Delta d_{t}\right\|_{L^{2}}^{2} .
\end{aligned}
$$

Now, differentiating 1.14 with respect to time variable $t$ yields

$$
d_{t t}-\Delta d_{t}=-(u \cdot \nabla d)_{t}+\left(|\nabla d|^{2} d\right)_{t}
$$


Multiplying (3.29) by $\Delta d_{t}$, and integrating the resulting equality over $\Omega$, we get

$$
\begin{aligned}
& \frac{1}{2} \frac{d}{d t} \int\left|\nabla d_{t}\right|^{2} d x+\int\left|\Delta d_{t}\right|^{2} d x \\
= & \int u_{t} \cdot \nabla d \cdot \Delta d_{t} d x+\int u \cdot \nabla d_{t} \cdot \Delta d_{t} d x-\int|\nabla d|^{2} d_{t} \cdot \Delta d_{t} d x \\
& -2 \int\left(\nabla d: \nabla d_{t}\right) d \cdot \Delta d_{t} d x \\
\leq & C \int\left|\nabla u_{t}\right||\nabla d|\left|\nabla d_{t}\right| d x+C \int\left|u_{t}\right|\left|\nabla^{2} d\right|\left|\nabla d_{t}\right| d x+C \int|\nabla d|^{2}\left|d_{t}\right|\left|\Delta d_{t}\right| d x \\
& +C \int|\nabla d|\left|\nabla d_{t}\right|\left|\Delta d_{t}\right| d x+C \int|u|\left|\nabla d_{t}\right|\left|\Delta d_{t}\right| d x \\
= & : \sum_{i=1}^{5} \mathcal{I}_{3 i} .
\end{aligned}
$$

By virtue of Hölder's, Poincaré's and Gagliardo-Nirenberg inequalities, (3.8), and (3.1), we have

$$
\begin{aligned}
\mathcal{I}_{31} & \leq C\left\|\nabla u_{t}\right\|_{L^{2}}\|\nabla d\|_{L^{4}}\left\|\nabla d_{t}\right\|_{L^{4}} \leq \delta \underline{\mu}\left\|\nabla u_{t}\right\|_{L^{2}}^{2}+C\|\nabla d\|_{L^{4}}^{2}\left\|\nabla d_{t}\right\|_{L^{4}}^{2} \\
& \leq \delta \underline{\mu}\left\|\nabla u_{t}\right\|_{L^{2}}^{2}+C\|\nabla d\|_{L^{2}}\|\Delta d\|_{L^{2}}\left\|\Delta d_{t}\right\|_{L^{2}}^{2} \leq \delta \underline{\mu}\left\|\nabla u_{t}\right\|_{L^{2}}^{2}+C M_{3}^{1 / 2}\|\nabla d\|_{L^{2}}\left\|\Delta d_{t}\right\|_{L^{2}}^{2}, \\
\mathcal{I}_{32} & \leq C\left\|u_{t}\right\|_{L^{2}}\left\|\nabla^{2} d\right\|_{L^{4}}\left\|\nabla d_{t}\right\|_{L^{4}} \leq C\left\|\nabla u_{t}\right\|_{L^{2}}\|\Delta d\|_{L^{2}}^{1 / 2}\left\|\nabla^{3} d\right\|_{L^{2}}^{1 / 2}\left\|\nabla d_{t}\right\|_{L^{2}}^{1 / 2}\left\|\Delta d_{t}\right\|_{L^{2}}^{1 / 2} \\
& \leq \delta \underline{\mu}\left\|\nabla u_{t}\right\|_{L^{2}}^{2}+C\|\Delta d\|_{L^{2}}\left\|\nabla^{3} d\right\|_{L^{2}}\left\|\nabla d_{t}\right\|_{L^{2}}\left\|\Delta d_{t}\right\|_{L^{2}} \\
& \leq \delta \underline{\mu}\left\|\nabla u_{t}\right\|_{L^{2}}^{2}+\kappa\left\|\Delta d_{t}\right\|_{L^{2}}^{2}+C\|\Delta d\|_{L^{2}}^{2}\left\|\nabla^{3} d\right\|_{L^{2}}^{2}\left\|\nabla d_{t}\right\|_{L^{2}}^{2} \\
& \leq \delta \underline{\mu}\left\|\nabla u_{t}\right\|_{L^{2}}^{2}+\kappa\left\|\Delta d_{t}\right\|_{L^{2}}^{2}+C M_{3}\left\|\nabla^{3} d\right\|_{L^{2}}^{2}\left\|\nabla d_{t}\right\|_{L^{2}}^{2}, \\
\mathcal{I}_{33} & \leq C\left\|\Delta d_{t}\right\|_{L^{2}}\|\nabla d\|_{L^{\infty}}^{2}\left\|d_{t}\right\|_{L^{2}} \leq C\left\|\Delta d_{t}\right\|_{L^{2}}\|\nabla d\|_{L^{2}}\left\|\nabla^{3} d\right\|_{L^{2}}\left\|\nabla d_{t}\right\|_{L^{2}} \\
& \leq \delta\left\|\Delta d_{t}\right\|_{L^{2}}^{2}+C\left\|\nabla^{3} d\right\|_{L^{2}}^{2}\left\|\nabla d_{t}\right\|_{L^{2}}^{2}, \\
\mathcal{I}_{34} & \leq C\left\|\Delta d_{t}\right\|_{L^{2}}\|\nabla d\|_{L^{4}}\left\|\nabla d_{t}\right\|_{L^{4}} \leq \delta\left\|\Delta d_{t}\right\|_{L^{2}}^{2}+C\|\nabla d\|_{L^{2}}^{2}\left\|\nabla^{2} d\right\|_{L^{2}}^{2}\left\|\nabla d_{t}\right\|_{L^{2}}^{2} \\
& \leq \delta\left\|\Delta d_{t}\right\|_{L^{2}}^{2}+C\left\|\nabla^{3} d\right\|_{L^{2}}^{2}\left\|\nabla d_{t}\right\|_{L^{2}}^{2}, \\
\mathcal{I}_{35} & \leq C\|u\|_{L^{\infty}}\left\|\nabla d_{t}\right\|_{L^{2}}\left\|\Delta d_{t}\right\|_{L^{2}} \leq \delta\left\|\Delta d_{t}\right\|_{L^{2}}^{2}+C\|\nabla u\|_{H^{1}}^{2}\left\|\nabla d_{t}\right\|_{L^{2}}^{2} .
\end{aligned}
$$

Thus, inserting the estimates of $\mathcal{I}_{3 i}(i=1,2, \ldots, 5)$ into 3.30 , we arrive at

$$
\begin{aligned}
\frac{1}{2} \frac{d}{d t}\left\|\nabla d_{t}\right\|_{L^{2}}^{2}+\left\|\Delta d_{t}\right\|_{L^{2}}^{2} \leq & \delta \underline{\mu}\left\|\nabla u_{t}\right\|_{L^{2}}^{2}+C\left(\|\nabla u\|_{H^{1}}^{2}+\left(M_{3}+1\right)\left\|\nabla^{3} d\right\|_{L^{2}}^{2}\right)\left\|\nabla d_{t}\right\|_{L^{2}}^{2} \\
& +(\delta+\kappa)\left\|\Delta d_{t}\right\|_{L^{2}}^{2}+C M_{3}^{1 / 2}\|\nabla d\|_{L^{2}}\left\|\Delta d_{t}\right\|_{L^{2}}^{2}
\end{aligned}
$$

Adding the resulting inequality with (3.31) and (3.28), taking $\delta$ and $\kappa$ suitable small, we 
get after using (3.20) and 3.21 that

$$
\begin{aligned}
& \frac{1}{2} \frac{d}{d t}\left(\left\|\sqrt{\rho} u_{t}\right\|_{L^{2}}^{2}+\left\|\nabla d_{t}\right\|_{L^{2}}^{2}\right)+\underline{\mu}\left\|\nabla u_{t}\right\|_{L^{2}}^{2}+\left\|\Delta d_{t}\right\|_{L^{2}}^{2} \\
\leq & C\left(M_{3}^{3 / 2}+1\right)^{2}\left(\|\nabla u\|_{H^{1}}^{2}+\left\|\nabla^{3} d\right\|_{L^{2}}^{2}\right)\left(\left\|\sqrt{\rho} u_{t}\right\|_{L^{2}}^{2}+\left\|\nabla d_{t}\right\|_{L^{2}}^{2}\right)+C M_{3}^{1 / 2}\|\nabla d\|_{L^{2}}\left\|\Delta d_{t}\right\|_{L^{2}}^{2} \\
\leq & C\left(M_{3}^{9 / 2}+1\right)^{3}\left(\left\|\sqrt{\rho} u_{t}\right\|_{L^{2}}^{2}+\left\|\nabla d_{t}\right\|_{L^{2}}^{2}\right)\left(\left\|\sqrt{\rho} u_{t}\right\|_{L^{2}}^{2}+\left\|\nabla d_{t}\right\|_{L^{2}}^{2}\right)+C M_{3}^{1 / 2}\|\nabla d\|_{L^{2}}\left\|\Delta d_{t}\right\|_{L^{2}}^{2} .
\end{aligned}
$$

Next, multiplying the above inequality by $t$, we get

$$
\begin{aligned}
& \frac{1}{2} \frac{d}{d t}\left[t\left(\left\|\sqrt{\rho} u_{t}\right\|_{L^{2}}^{2}+\left\|\nabla d_{t}\right\|_{L^{2}}^{2}\right)\right]+t\left(\underline{\mu}\left\|\nabla u_{t}\right\|_{L^{2}}^{2}+\left\|\Delta d_{t}\right\|_{L^{2}}^{2}\right) \\
\leq & C_{6}\left(M_{3}^{9 / 2}+1\right)^{3}\left(\left\|\sqrt{\rho} u_{t}\right\|_{L^{2}}^{2}+\left\|\nabla d_{t}\right\|_{L^{2}}^{2}\right)\left[t\left(\left\|\sqrt{\rho} u_{t}\right\|_{L^{2}}^{2}+\left\|\nabla d_{t}\right\|_{L^{2}}^{2}\right)\right] \\
& +C_{6} M_{3}^{1 / 2}\|\nabla d\|_{L^{2}}\left[t\left\|\Delta d_{t}\right\|_{L^{2}}^{2}\right]+\left\|\sqrt{\rho} u_{t}\right\|_{L^{2}}^{2}+\left\|\nabla d_{t}\right\|_{L^{2}}^{2},
\end{aligned}
$$

which, integrating over $[0, \zeta(T)]$, using Gronwall's inequality and (3.9) lead to

$$
\sup _{0 \leq t \leq \zeta(T)}\left[t\left(\left\|\sqrt{\rho} u_{t}\right\|_{L^{2}}^{2}+\left\|\nabla d_{t}\right\|_{L^{2}}^{2}\right)\right]+\int_{0}^{\zeta(T)} t\left(\underline{\mu}\left\|\nabla u_{t}\right\|_{L^{2}}^{2}+\left\|\Delta d_{t}\right\|_{L^{2}}^{2}\right) d t \leq 2 M_{3},
$$

provided $\sup _{0 \leq t \leq T}\|\nabla d\|_{L^{2}} \leq M_{0} \leq \epsilon_{6}:=\min \left\{\epsilon_{5}, \frac{1}{2 C_{6} M_{3}^{1 / 2}}\right\}$.

Similarly, multiplying (3.32) by $e^{\sigma t}$ and integrating over $[\zeta(T), T]$, by Gronwall's inequality, (3.33), and (3.10), we get

$$
\begin{aligned}
& \sup _{\zeta(T) \leq t \leq T}\left[e^{\sigma t}\left(\left\|\sqrt{\rho} u_{t}\right\|_{L^{2}}^{2}+\left\|\nabla d_{t}\right\|_{L^{2}}^{2}\right)\right]+\int_{\zeta(T)}^{T} e^{\sigma t}\left(\underline{\mu}\left\|\nabla u_{t}\right\|_{L^{2}}^{2}+\left\|\Delta d_{t}\right\|_{L^{2}}^{2}\right) d t \\
\leq & C\left(M_{3}^{9 / 4}+1\right)^{2} \exp \left\{e^{-\zeta(T)} \int_{\zeta(T)}^{T} e^{\sigma t}\left(\left\|\sqrt{\rho} u_{t}\right\|_{L^{2}}^{2}+\left\|\nabla d_{t}\right\|_{L^{2}}^{2}\right) d t\right\} \\
& \times\left\{\left.e^{\sigma(t)}\left(\left\|\sqrt{\rho} u_{t}\right\|_{L^{2}}^{2}+\left\|\nabla d_{t}\right\|_{L^{2}}^{2}\right)\right|_{t=\zeta(T)}+\int_{\zeta(T)}^{T} e^{\sigma t}\left(\left\|\sqrt{\rho} u_{t}\right\|_{L^{2}}^{2}+\left\|\nabla d_{t}\right\|_{L^{2}}^{2}\right) d t\right\} \\
\leq & C M_{3}^{2}\left(M_{3}^{9 / 2}+1\right)^{3},
\end{aligned}
$$

provided $\sup _{0 \leq t \leq T}\|\nabla d\|_{L^{2}} \leq M_{0} \leq \epsilon_{6}:=\min \left\{\epsilon_{5}, \frac{1}{2 C_{6} M_{3}^{1 / 2}}\right\}$.

Finally, it follows from $(3.20),(3.21),(3.10)$, and 3.24$)$ that $(3.25)$ holds. The proof of Lemma 3.4 is completed.

We will use Lemmas 3.23 .4 to prove the following time-independent bound on the $L^{1}\left(0, T ; L^{\infty}\right)$-norm of $\nabla u$ which is important for obtaining the uniform one (with respect to time) on the $L^{\infty}\left(0, T ; L^{q}\right)$-norm of the gradient of $\mu(\rho)$. 
Lemma 3.5. Let $(\rho, u, d, P)$ be a smooth solution to (1.1)-1.4 satisfying (3.1). Then there exists a generic positive constant $C$ depending only on $\Omega, q, \underline{\mu}, \bar{\mu}, M_{1}, M_{2}$, and $M_{3}$ such that

$$
\begin{aligned}
\int_{0}^{T}\|\nabla u\|_{L^{\infty}} \leq & C \bar{\rho}^{3 / 4} M_{3}\left(M_{3}^{9 / 2}+1\right)^{3 / 2}+C \bar{\rho}^{(6 r-6) / r} M_{3}^{\frac{5 r-6}{2 r}} M_{2} \\
& +C \sup _{0 \leq t \leq T}\|\nabla d\|_{L^{2}}^{5 / 6} M_{3}^{7 / 12}\left(M_{3}^{9 / 2}+1\right)^{7 / 6} .
\end{aligned}
$$

Proof. First, it follows from Lemma 2.2, (3.1), (3.4), Gagliardo-Nirenberg and Hölder's inequalities that for $r \in(2, \min \{q, 3\})$,

$$
\begin{aligned}
\|\nabla u\|_{W^{1, r}} \leq & C\|F\|_{L^{r}}\left(1+\|\nabla \mu(\rho)\|_{L^{q}}\right)^{\frac{q r}{2(q-r)}} \\
\leq & C\left\|\rho u_{t}\right\|_{L^{3}}+C\|\rho u \cdot \nabla u\|_{L^{r}}+C\|\mid \nabla d\| \nabla^{2} d\|\|_{L^{3}} \\
\leq & C\left\|\rho u_{t}\right\|_{L^{2}}^{1 / 2}\left\|\rho u_{t}\right\|_{L^{6}}^{1 / 2}+C \bar{\rho}\|u\|_{L^{3}}\|\nabla u\|_{L^{3 r /(3-r)}}+C\|\nabla d\|_{L^{\infty}}\left\|\nabla^{2} d\right\|_{L^{3}} \\
\leq & C \bar{\rho}^{3 / 4}\left\|\sqrt{\rho} u_{t}\right\|_{L^{2}}^{1 / 2}\left\|\nabla u_{t}\right\|_{L^{2}}^{1 / 2}+C \bar{\rho}\|\nabla u\|_{L^{2}}^{\frac{7 r-6}{6 r-6}}\|\nabla u\|_{W^{1, r}}^{\frac{5 r-6}{6 r-6}} \\
& +C\|\nabla d\|_{L^{\infty}}\left\|\nabla^{3} d\right\|_{L^{2}}^{2 / 3}\|\nabla d\|_{L^{2}}^{1 / 3} \\
\leq & C \bar{\rho}^{3 / 4}\left\|\sqrt{\rho} u_{t}\right\|_{L^{2}}^{1 / 2}\left\|\nabla u_{t}\right\|_{L^{2}}^{1 / 2}+C \bar{\rho}^{(6 r-6) / r}\|\nabla u\|_{L^{2}}^{(7 r-6) / r} \\
& +\frac{1}{2}\|\nabla u\|_{W^{1, r}}+C\|\nabla d\|_{L^{2}}^{5 / 6}\left\|\nabla^{3} d\right\|_{L^{2}}^{7 / 6},
\end{aligned}
$$

which directly deduces that

$$
\|\nabla u\|_{W^{1, r}} \leq C \bar{\rho}^{3 / 4}\left\|\sqrt{\rho} u_{t}\right\|_{L^{2}}^{1 / 2}\left\|\nabla u_{t}\right\|_{L^{2}}^{1 / 2}+C \bar{\rho}^{(6 r-6) / r}\|\nabla u\|_{L^{2}}^{(7 r-6) / r}+C\|\nabla d\|_{L^{2}}^{5 / 6}\left\|\nabla^{3} d\right\|_{L^{2}}^{7 / 6} .
$$

On the one hand, it follows from 3.23, 3.5, 3.21), and 3.9 that for $t \in[0, \zeta(T)]$,

$$
\begin{aligned}
& \int_{0}^{\zeta(T)}\|\nabla u\|_{L^{\infty}} d t \\
\leq & C \bar{\rho}^{3 / 4}\left[\int_{0}^{\zeta(T)} t^{-1 / 2}\left\|\sqrt{\rho} u_{t}\right\|_{L^{2}}^{2 / 3} d t\right]^{3 / 4} \cdot\left[\int_{0}^{\zeta(T)} t^{3 / 2}\left\|\nabla u_{t}\right\|_{L^{2}}^{2} d t\right]^{1 / 4} \\
& +C\left(M_{3}^{9 / 2}+1\right)^{7 / 6} \sup _{0 \leq t \leq \zeta(T)}\|\nabla d\|_{L^{2}}^{5 / 6} \\
& \times \sup _{0 \leq t \leq \zeta(T)}\left(t\left\|\sqrt{\rho} u_{t}\right\|_{L^{2}}^{2}+t\left\|\nabla d_{t}\right\|_{L^{2}}^{2}\right)^{7 / 12} \int_{0}^{\zeta(T)} t^{-1+5 / 7} d t \\
& +C \bar{\rho}^{(6 r-6) / r} \sup _{0 \leq t \leq \zeta(T)}\left(\|\nabla u\|_{L^{2}}^{2}\right)^{\frac{5 r-6}{2 r}} \int_{0}^{\zeta(T)}\|\nabla u\|_{L^{2}}^{2} d t \\
\leq & C \bar{\rho}^{3 / 4} M_{3}^{1 / 4}\left[\int_{0}^{\zeta(T)} t^{-1 / 2} \cdot t^{-\frac{1}{2} \cdot \frac{2}{3}} d t\right]^{3 / 4} \cdot\left[\int_{0}^{\zeta(T)} t^{3 / 2}\left\|\nabla u_{t}\right\|_{L^{2}}^{2} d t\right]^{1 / 4}
\end{aligned}
$$




$$
\begin{aligned}
& +C \bar{\rho}^{(6 r-6) / r} M_{3}^{\frac{5 r-6}{2 r}} M_{2}+C \sup _{0 \leq t \leq \zeta(T)}\|\nabla d\|_{L^{2}}^{5 / 6} M_{3}^{7 / 12}\left(M_{3}^{9 / 2}+1\right)^{7 / 6} \\
\leq & C \bar{\rho}^{3 / 4} M_{3}^{1 / 2}+C \bar{\rho}^{(6 r-6) / r} M_{3}^{\frac{5 r-6}{2 r}} M_{2}+C \sup _{0 \leq t \leq \zeta(T)}\|\nabla d\|_{L^{2}}^{5 / 6} M_{3}^{7 / 12}\left(M_{3}^{9 / 2}+1\right)^{7 / 6} .
\end{aligned}
$$

On the other hand, using (3.10, 3.24), 3.21) and (3.5), we obtain that for $t \in[\zeta(T), T]$,

$$
\begin{aligned}
\int_{\zeta(T)}^{T}\|\nabla u\|_{L^{\infty}} d t \leq & C \bar{\rho}^{(6 r-6) / r} \sup _{\zeta(T) \leq t \leq T}\left(e^{\sigma t}\|\nabla u\|_{L^{2}}^{2}\right)^{\frac{5 r-6}{2 r}} e^{-\frac{(5 r-6) \sigma \zeta(T)}{2 r}} \int_{\zeta(T)}^{T}\|\nabla u\|_{L^{2}}^{2} d t \\
& +C \sup _{0 \leq t \leq \zeta(T)}\|\nabla d\|_{L^{2}}^{5 / 6}\left[\int_{\zeta(T)}^{T} e^{\sigma t}\left\|\nabla^{3} d\right\|_{L^{2}}^{2} d t\right]^{7 / 12}\left[\int_{\zeta(T)}^{T} e^{-\frac{7}{5} \sigma t} d t\right]^{5 / 12} \\
& +C \bar{\rho}^{3 / 4} \int_{\zeta(T)}^{T}\left(\left\|\sqrt{\rho} u_{t}\right\|_{L^{2}}+\left\|\nabla u_{t}\right\|_{L^{2}}\right) d t \\
\leq & C \bar{\rho}^{3 / 4} \sup _{\zeta(T) \leq t \leq T}\left(e^{\sigma t}\left\|\sqrt{\rho} u_{t}\right\|_{L^{2}}^{2}\right)^{1 / 2} \int_{\zeta(T)}^{T} e^{-\frac{\sigma t}{2}} d t+C \bar{\rho}^{(6 r-6) / r} M_{3}^{\frac{5 r-6}{2 r}} M_{2} \\
& +C \bar{\rho}^{3 / 4}\left(\int_{\zeta(T)}^{T} e^{-\sigma t} d t\right)^{1 / 2}\left(\int_{\zeta(T)}^{T} e^{\sigma t}\left\|\nabla u_{t}\right\|_{L^{2}}^{2} d t\right)^{1 / 2} \\
& +C \sup _{\zeta(T) \leq t \leq T}\|\nabla d\|_{L^{2}}^{5 / 6} M_{3}^{7 / 12}\left(M_{3}^{9 / 2}+1\right)^{7 / 6} \\
\leq & C \bar{\rho}^{3 / 4} M_{3}\left(M_{3}^{9 / 2}+1\right)^{3 / 2}+C \bar{\rho}^{(6 r-6) / r} M_{3}^{\frac{5 r-6}{2 r}} M_{2} \\
& +C \sup _{\zeta(T) \leq t \leq T}\|\nabla d\|_{L^{2}}^{5 / 6} M_{3}^{7 / 12}\left(M_{3}^{9 / 2}+1\right)^{7 / 6} \cdot
\end{aligned}
$$

Combining this with (3.36) gives (3.34) and finishes the proof of Lemma 3.5 .

With Lemmas 3.23 .5 at hand, we are in a position to prove Proposition 3.1 .

Proof of Proposition 3.1. First, it follows from $(1.1)_{1}$ that

$$
\frac{d}{d t}\|\nabla \mu(\rho)\|_{L^{q}} \leq C\|\nabla u\|_{L^{\infty}}\|\nabla \mu(\rho)\|_{L^{q}},
$$

which together with Gronwall's inequality and (3.34) yields

$$
\begin{aligned}
& \sup _{0 \leq t \leq T}\|\nabla \mu(\rho)\|_{L^{q}} \\
& \leq\left\|\nabla \mu\left(\rho_{0}\right)\right\|_{L^{q}} \exp \left\{C \int_{0}^{T}\|\nabla u\|_{L^{\infty}} d t\right\} \\
& \leq\left\|\nabla \mu\left(\rho_{0}\right)\right\|_{L^{q}} \exp \left\{C_{7} \bar{\rho}^{3 / 4} M_{3}\left(M_{3}^{9 / 2}+1\right)^{3 / 2}+C_{7} \bar{\rho}^{(6 r-6) / r} M_{3}^{\frac{5 r-6}{2 r}} M_{2}\right. \\
&\left.+C_{7} \sup _{0 \leq t \leq T}\|\nabla d\|_{L^{2}}^{5 / 6} M_{3}^{7 / 12}\left(M_{3}^{9 / 2}+1\right)^{7 / 6}\right\}
\end{aligned}
$$

$\leq 2 M_{1}$, 
provided $\sup _{0 \leq t \leq T}\left(\bar{\rho}+\|\nabla d\|_{L^{2}}\right) \leq M_{0} \leq \epsilon_{7}:=\min \left\{\epsilon_{6},\left(\frac{\ln 2}{3 C_{7} M_{3}\left(M_{3}^{9 / 2}+1\right)^{3 / 2}}\right)^{4 / 3}\right.$, $\left.\left(\frac{\ln 2}{3 C_{7} M_{3}^{(5 r-6) /(2 r)} M_{2}}\right)^{r /(6 r-6)},\left(\frac{\ln 2}{3 C_{7} M_{3}^{7 / 12}\left(M_{3}^{9 / 2}+1\right)^{7 / 6}}\right)^{4 / 3}\right\}$.

Next, we deduce from $(3.22$ ) and 3.5 that

$$
\begin{aligned}
& \sup _{0 \leq t \leq T} e^{\sigma t}\left(\underline{\mu}\|\nabla u\|_{L^{2}}^{2}+\|\Delta d\|_{L^{2}}^{2}\right)+\int_{0}^{T} e^{\sigma t}\left(\left\|\sqrt{\rho} u_{t}\right\|_{L^{2}}^{2}+\left\|\nabla d_{t}\right\|_{L^{2}}^{2}\right) d t \\
\leq & C \sup _{0 \leq t \leq T} e^{\sigma t}\left[\|\nabla d\|_{L^{2}}^{2}\|\Delta d\|_{L^{2}}^{2}\right]+\int_{0}^{T} e^{\sigma t}\left(\bar{\mu}\|\nabla u\|_{L^{2}}^{2}+\|\Delta d\|_{L^{2}}^{2}\right) d t+M_{3} \\
& +\sup _{0 \leq t \leq T} \frac{\underline{\mu}}{\overline{2}}\left[e^{\sigma t}\|\nabla u\|_{L^{2}}^{2}\right]+C \sup _{0 \leq t \leq T}\|\nabla d\|_{L^{2}} \int_{0}^{T} e^{\sigma t}\left(\|\Delta d\|_{L^{2}}^{2}+\underline{\mu}\|\nabla u\|_{L^{2}}^{2}\right) d t \\
\leq & C \sup _{0 \leq t \leq T} e^{\sigma t}\left[\|\nabla d\|_{L^{3}}^{2}\|\Delta d\|_{L^{2}}^{2}\right]+2\left(\frac{\bar{\mu}}{\underline{\mu}}+1\right) M_{2}+M_{3} \\
& +\sup _{0 \leq t \leq T} \frac{\underline{\mu}}{2}\left[e^{\sigma t}\|\nabla u\|_{L^{2}}^{2}\right]+C \sup _{0 \leq t \leq T}\|\nabla d\|_{L^{2}}\left(\left\|\sqrt{\rho_{0}} u_{0}\right\|_{L^{2}}^{2}+\left\|\nabla d_{0}\right\|_{L^{2}}^{2}\right) \\
\leq & C_{8} \sup _{0 \leq t \leq T} e^{\sigma t}\left[\|\nabla d\|_{L^{2}}^{2}\|\Delta d\|_{L^{2}}^{2}\right]+2\left(\frac{\bar{\mu}}{\mu}+1\right) M_{2}+M_{3} \\
& +\sup _{0 \leq t \leq T} \frac{\mu}{\overline{2}}\left[e^{\sigma t}\|\nabla u\|_{L^{2}}^{2}\right]+C_{8} \sup _{0 \leq t \leq T}\|\nabla d\|_{L^{2}} M_{3}
\end{aligned}
$$

which leads to

$$
\sup _{0 \leq t \leq T} e^{\sigma t}\left(\underline{\mu}\|\nabla u\|_{L^{2}}^{2}+\|\Delta d\|_{L^{2}}^{2}\right) \leq 2\left[2\left(\frac{\bar{\mu}}{\underline{\mu}}+1\right) M_{2}+\frac{3}{2} M_{3}\right],
$$

provided $\sup _{0 \leq t \leq T}\|\nabla d\|_{L^{2}} \leq M_{0} \leq \epsilon_{8}:=\min \left\{\epsilon_{7}, \frac{1}{2 C_{8}}\right\}$.

Moreover, it remains ro prove $\sup _{0 \leq t \leq T}\|\nabla d\|_{L^{2}} \leq M_{0} / 4$. In fact, multiplying (1.1) 4 by $-\Delta d$ and integrating by parts over $\Omega$, it follows that

$$
\begin{aligned}
\frac{1}{2} \frac{d}{d t}\|\nabla d\|_{L^{2}}^{2}+\|\Delta d\|_{L^{2}}^{2} & \leq C \int|\nabla u\|\nabla d\| \nabla d| d x+C \int|\nabla d|^{2}\left|\nabla^{2} d\right| d x \\
& \leq C\|\nabla u\|_{L^{\infty}}\|\nabla d\|_{L^{2}}^{2}+C\|\nabla d\|_{L^{4}}^{2}\|\Delta d\|_{L^{2}} \\
& \leq C\|\nabla u\|_{L^{\infty}}\|\nabla d\|_{L^{2}}^{2}+C_{9}\|\nabla d\|_{L^{2}}\|\Delta d\|_{L^{2}}^{2},
\end{aligned}
$$

that is

$$
\frac{d}{d t}\|\nabla d\|_{L^{2}}^{2}+\|\Delta d\|_{L^{2}}^{2} \leq C\|\nabla u\|_{L^{\infty}}\|\nabla d\|_{L^{2}}^{2}
$$

provided $\sup _{0 \leq t \leq T}\|\nabla d\|_{L^{2}} \leq M_{0} \leq \epsilon_{9}=\min \left\{\epsilon_{8}, \frac{1}{2 C_{9}}\right\}$. Then, 3.41 together with 3.34 and Gronwall's inequality leads to

$$
\begin{aligned}
\sup _{0 \leq t \leq T}\|\nabla d\|_{L^{2}} & \leq C_{10}\left\{M_{3}^{1 / 2}\left(M_{3}^{9 / 2}+1\right)^{3 / 4}+M_{3}^{\frac{5 r-6}{4 r}} M_{2}+M_{3}^{7 / 24}\left(M_{3}^{9 / 2}+1\right)^{7 / 12}\right\}\left\|\nabla d_{0}\right\|_{L^{2}} \\
& \leq \frac{M_{0}}{4}
\end{aligned}
$$


provided $M_{0} \leq \epsilon_{10}:=\min \left\{\epsilon_{9}, \frac{1}{12 C_{10} M_{3}^{1 / 2}\left(M_{3}^{9 / 2}+1\right)^{3 / 4}}, \frac{1}{12 C_{10} M_{3}^{(5 r-6) /(4 r)} M_{2}}\right.$, $\left.\frac{1}{12 C_{10} M_{3}^{7 / 24}\left(M_{3}^{9 / 2}+1\right)^{7 / 12}}\right\}$.

Finally, choosing $\epsilon_{0}:=\min \left\{\epsilon_{10}, \epsilon / 4\right\}$, we directly obtain $(3.2)$ from $(3.4),(3.39)$, and (3.42). The proof of Proposition 3.1 is finished.

The following Lemma 3.6 is necessary for further estimates on the higher-order derivatives of the strong solution $(\rho, u, d, P)$.

Lemma 3.6. Let $(\rho, u, d, P)$ be a smooth solution to (1.1)-1.4 satisfying (3.1). Then there exists a generic positive constant $C$ depending only on $\Omega, q, \underline{\mu}, \bar{\mu}, M_{1}, M_{2}$, and $M_{3}$ such that for $r \in(2, \min \{q, 3\})$,

$$
\begin{aligned}
& \sup _{0 \leq t \leq T}\|\rho\|_{H^{1} \cap W^{1, q}}+\sup _{0 \leq t \leq T} e^{\sigma t}\left(\zeta\|\nabla u\|_{H^{1}}^{2}+\zeta\|P\|_{H^{1}}^{2}+\zeta\left\|\nabla d_{t}\right\|_{L^{2}}^{2}+\zeta\left\|\nabla^{3} d\right\|_{L^{2}}^{2}\right) \\
& +\int_{0}^{T} e^{\sigma t}\left(\|\nabla u\|_{H^{1}}^{2}+\|P\|_{H^{1}}^{2}+\left\|\nabla^{3} d\right\|_{L^{2}}^{2}+\zeta^{7 / 6}\|\nabla u\|_{W^{1, r}}^{2}+\zeta^{7 / 6}\|P\|_{W^{1, r}}^{2}+\zeta^{2}\left\|\nabla^{4} d\right\|_{L^{2}}^{2}\right) d t \\
& +\int_{0}^{T} \zeta e^{\sigma t}\left(\left\|\nabla u_{t}\right\|_{L^{2}}^{2}+\left\|\Delta d_{t}\right\|_{L^{2}}^{2}\right) d t \leq C .
\end{aligned}
$$

Proof. First, similar to 3.38 and 3.39 , we have

$$
\|\nabla \rho\|_{L^{2} \cap L^{q}} \leq 2\left\|\nabla \rho_{0}\right\|_{L^{2} \cap L^{q}} .
$$

Next, multiplying (3.32) by $\zeta e^{\sigma t}$, we get after using Gronwall's inequality, (3.9), 3.10), 3.23 , and 3.24 that

$$
\sup _{0 \leq t \leq T} \zeta e^{\sigma t}\left(\left\|\sqrt{\rho} u_{t}\right\|_{L^{2}}^{2}+\left\|\nabla d_{t}\right\|_{L^{2}}^{2}\right)+\int_{0}^{T} \zeta e^{\sigma t}\left(\left\|\nabla u_{t}\right\|_{L^{2}}^{2}+\left\|\Delta d_{t}\right\|_{L^{2}}^{2}\right) d t \leq C .
$$

Combining this, $3.20,3.21$, and 3.40 gives

$$
\begin{aligned}
& \sup _{0 \leq t \leq T} e^{\sigma t}\left(\zeta\|\nabla u\|_{H^{1}}^{2}+\zeta\|P\|_{H^{1}}^{2}+\zeta\left\|\nabla d_{t}\right\|_{L^{2}}^{2}+\zeta\left\|\nabla^{3} d\right\|_{L^{2}}^{2}\right) \\
& +\int_{0}^{T} e^{\sigma t}\left(\|\nabla u\|_{H^{1}}^{2}+\|P\|_{H^{1}}^{2}+\left\|\nabla^{3} d\right\|_{L^{2}}^{2}\right) d t \leq C .
\end{aligned}
$$

Moreover, by $H^{4}$-estimate of 1.14 , using Poincaré's inequality, we have

$$
\begin{aligned}
\left\|\nabla^{4} d\right\|_{L^{2}} \leq & C\left\|d_{t}\right\|_{H^{2}}+C\|u \cdot \nabla d\|_{H^{2}}+C\left\|\left.|| \nabla d\right|^{2} d\right\|_{H^{2}} \\
\leq & C\left\|d_{t}\right\|_{H^{2}}+C\left\||u|\left(|\nabla d|+\left|\nabla^{2} d\right|+\left|\nabla^{3} d\right|\right)\right\|_{L^{2}}+C\left\||\nabla u|\left(|\nabla d|+\left|\nabla^{2} d\right|\right)\right\|_{L^{2}} \\
& +C\left\|\left|\nabla^{2} u\left\|\left.\nabla d\left|\left\|_{L^{2}}+C\right\|\right| \nabla d\right|^{2}\right\|_{L^{2}}+C\left\||\nabla d|\left|\nabla^{2} d\right|\right\|_{L^{2}}+C\left\|\left|\nabla^{2} d\right|^{2}\right\|_{L^{2}}\right.\right. \\
& +C\left\||\nabla d|\left|\nabla^{3} d\right|\right\|_{L^{2}}
\end{aligned}
$$




$$
\begin{aligned}
\leq & C\left\|d_{t}\right\|_{H^{2}}+C\|u\|_{L^{\infty}}\|\nabla d\|_{H^{2}}+C\|\nabla d\|_{L^{\infty}}\|\nabla u\|_{H^{1}}+C\|\nabla u\|_{H^{1}}\left\|\nabla^{3} d\right\|_{L^{2}} \\
& +C\|\nabla d\|_{L^{\infty}}\|\nabla d\|_{H^{2}}+C\left\|\nabla^{2} d\right\|_{H^{1}}^{2} \\
\leq & C\left\|\Delta d_{t}\right\|_{L^{2}}+C\left(\left\|\nabla^{3} d\right\|_{L^{2}}+\|\nabla u\|_{H^{1}}\right)\left\|\nabla^{3} d\right\|_{L^{2}}+C\left\|\nabla^{3} d\right\|_{L^{2}}^{2}
\end{aligned}
$$

which along with 3.45 , and 3.46 leads to

$$
\int_{0}^{T} \zeta^{2} e^{\sigma t}\left\|\nabla^{4} d\right\|_{L^{2}}^{2} d t \leq C .
$$

Finally, it follows from 3.20$),(3.21),(3.35),(3.40)$, and $(3.4)$ that for $r \in(2, \min \{q, 3\})$,

$$
\begin{aligned}
\|\nabla u\|_{H^{1} \cap W^{1, r}}+\|P\|_{H^{1} \cap W^{1, r}} & \leq C\left\|\nabla u_{t}\right\|_{L^{2}}+C\|\nabla u\|_{L^{2}}+C\left\|\nabla^{3} d\right\|_{L^{2}}^{7 / 6} \\
& \leq C\left\|\nabla u_{t}\right\|_{L^{2}}+C\|\nabla u\|_{L^{2}}+C\left(\left\|\sqrt{\rho} u_{t}\right\|_{L^{2}}+\left\|\nabla d_{t}\right\|_{L^{2}}\right)^{7 / 6}
\end{aligned}
$$

which together with 3.45 and 3.5 implies

$$
\int_{0}^{T} \zeta^{7 / 6} e^{\sigma t}\left(\|\nabla u\|_{H^{1} \cap W^{1, r}}^{2}+\|P\|_{H^{1} \cap W^{1, r}}^{2}\right) d t \leq C .
$$

This combined with $3.45-(3.46)$ gives 3.43 and completed the proof of Lemma 3.6.

\section{Proof of Theorem 1.1}

With all the a priori estimates obtained in Section 3 at hand, we are now in a position to prove Theorem 1.1

Proof of Theorem 1.1. First, by Lemma 2.1, there exists a $T_{*}>0$ such that the initial boundary value problem (1.1) - (1.4) has a unique local strong solution $(\rho, u, d, P)$ on $\Omega \times$ $\left(0, T_{*}\right]$. It follows from $(1.6)$ that there exists a $T_{1} \in\left(0, T_{*}\right]$ such that (3.1) holds for $T=T_{1}$.

Next, set

$$
T^{*}:=\sup \{T \mid(\rho, u, d, P) \text { is a strong solution on } \Omega \times(0, T] \text { and (3.1) holds }\} .
$$

First, for any $0<\tau<T \leq T^{*}$, one deduces from $(3.2),(3.5)$, and $(3.43)$ that for any $q>2$,

$$
\nabla u, \nabla d, \Delta d \in C\left([\tau, T] ; L^{2} \cap L^{q}\right)
$$

where one has used the standard embedding theory

$$
L^{\infty}\left(\tau, T ; H^{1}\right) \cap H^{1}\left(\tau, T ; H^{-1}\right) \hookrightarrow C\left([\tau, T] ; L^{q_{0}}\right) \quad \text { for any } q_{0} \in[2, \infty) .
$$


Moreover, it follows from (3.1), 3.4), 3.44), and [21, Lemma 2.3] that

$$
\rho \in C\left([0, T] ; H^{1} \cap W^{1, q}\right), \quad \nabla \mu(\rho) \in C\left([0, T] ; L^{q}\right) .
$$

Now, we claim that

$$
T^{*}=\infty .
$$

Otherwise $T^{*}<\infty$. Proposition 3.1 implies that $(3.2)$ holds at $T=T^{*}$. It follows from (3.2), (3.5), (4.2), and (4.3) that

$$
\left(\rho^{*}, u^{*}, d^{*}\right)(x):=(\rho, u, d)\left(x, T^{*}\right)=\lim _{t \rightarrow T^{*}}(\rho, u, d)(x, t)
$$

satisfies

$$
\rho^{*} \in H^{1} \cap W^{1, q}, \quad u^{*} \in H_{0, \sigma}^{1}, \quad d^{*} \in H^{2} .
$$

Therefore, one can take $\left(\rho^{*}, \rho^{*} u^{*}, d^{*}\right)$ as the initial data and apply Lemma 2.1 to extend the local strong solution beyond $T^{*}$. This contradicts the assumption of $T^{*}$ in (4.1). Hence, (4.4) holds. We thus finish the proof of Theorem 1.1 since $(1.8)$ and $(1.9)$ follow directly from (3.44), and (3.43), respectively.

\section{Acknowledgments}

The author was supported by the Postdoctoral Research Foundation of Jiangsu Province (No. 2018K183C), Postdoctoral Science Foundation of China (No. 2018M642202), National Natural Science Foundation of China (No. 11901288).

\section{References}

[1] S. Ding, J. Huang and F. Xia, Global existence of strong solutions for incompressible hydrodynamic flow of liquid crystals with vacuum, Filomat 27 (2013), no. 7, 12471257.

[2] J. L. Ericksen, Hydrostatic theory of liquid crystals, Arch. Rational Mech. Anal. 9 (1962), 371-378.

[3] J. Gao, Q. Tao and Z.-a. Yao, Strong solutions to the density-dependent incompressible nematic liquid crystal flows, J. Differential Equations 260 (2016), no. 4, 3691-3748.

[4] C. He, J. Li and B. Lü, On the Cauchy problem of $3 D$ nonhomogeneous Navier-Stokes equations with density-dependent viscosity and vacuum, arXiv:1709.05608.

[5] J. L. Hineman and C. Wang, Well-posedness of nematic liquid crystal flow in $L_{\text {uloc }}^{3}\left(\mathbb{R}^{3}\right)$, Arch. Ration. Mech. Anal. 210 (2013), no. 1, 177-218. 
[6] D. Hoff, Compressible flow in a half-space with Navier boundary conditions, J. Math. Fluid Mech. 7 (2005), no. 3, 315-338.

[7] X. Hu and D. Wang, Global solution to the three-dimensional incompressible flow of liquid crystals, Comm. Math. Phys. 296 (2010), no. 3, 861-880.

[8] J. Huang, C. Wang and H. Wen, Time decay rate of global strong solutions to nematic liquid crystal flows in $\mathbb{R}_{+}^{3}$, J. Differential Equations 267 (2019), no. 3, 1767-1804.

[9] T. Huang and C. Wang, Blow up criterion for nematic liquid crystal flows, Comm. Partial Differential Equations 37 (2012), no. 5, 875-884.

[10] X. Huang, J. Li and Z. Xin, Global well-posedness of classical solutions with large oscillations and vacuum to the three-dimensional isentropic compressible Navier-Stokes equations, Comm. Pure Appl. Math. 65 (2012), no. 4, 549-585.

[11] X. Huang and Y. Wang, Global strong solution with vacuum to the two dimensional density-dependent Navier-Stokes system, SIAM J. Math. Anal. 46 (2014), no. 3, 17711788.

[12] F. M. Leslie, Some constitutive equations for liquid crystals, Arch. Rational Mech. Anal. 28 (1968), no. 4, 265-283.

[13] J. Li, Liquid crystal equations with infinite energy local well-posedness and blow up criterion, arXiv:1309.0072.

[14]_ Global strong and weak solutions to inhomogeneous nematic liquid crystal flow in two dimensions, Nonlinear Anal. 99 (2014), 80-94.

[15] _ Global strong solutions to the inhomogeneous incompressible nematic liquid crystal flow, Methods Appl. Anal. 22 (2015), no. 2, 201-220.

[16] _ Local existence and uniqueness of strong solutions to the Navier-Stokes equations with nonnegative density, J. Differential Equations 263 (2017), no. 10, 65126536.

[17] L. Li, Q. Liu and X. Zhong, Global strong solution to the two-dimensional densitydependent nematic liquid crystal flows with vacuum, Nonlinearity 30 (2017), no. 11, 4062-4088.

[18] X. Li, Global strong solution for the incompressible flow of liquid crystals with vacuum in dimension two, Discrete Contin. Dyn. Syst. 37 (2017), no. 9, 4907-4922. 
[19] X. Li and D. Wang, Global strong solution to the density-dependent incompressible flow of liquid crystals, Trans. Amer. Math. Soc. 367 (2015), no. 4, 2301-2338.

[20] F. Lin and C. Wang, Global existence of weak solutions of the nematic liquid crystal flow in dimension three, Comm. Pure Appl. Math. 69 (2016), no. 8, 1532-1571.

[21] P.-L. Lions, Mathematical Topics in Fluid Mechanics I: Incompressible Models, Oxford Lecture Series in Mathematics and its Applications 3, Oxford Science Publications, The Clarendon Press, Oxford University Press, New York, 1996.

[22] Q. Liu, S. Liu, W. Tan and X. Zhong, Global well-posedness of the 2D nonhomogeneous incompressible nematic liquid crystal flows, J. Differential Equations 261 (2016), no. 11, 6521-6569.

[23] S. Liu and J. Zhang, Global well-posedness for the two-dimensional equations of nonhomogeneous incompressible liquid crystal flows with nonnegative density, Discrete Contin. Dyn. Syst. Ser. B 21 (2016), no. 8, 2631-2648.

[24] B. Lü and S. Song, On local strong solutions to the three-dimensional nonhomogeneous Navier-Stokes equations with density-dependent viscosity and vacuum, Nonlinear Anal. Real World Appl. 46 (2019), 58-81.

[25] C. Wang, Well-posedness for the heat flow of harmonic maps and the liquid crystal flow with rough initial data, Arch. Ration. Mech. Anal. 200 (2011), no. 1, 1-19.

[26] H. Wen and S. Ding, Solutions of incompressible hydrodynamic flow of liquid crystals, Nonlinear Anal. Real World Appl. 12 (2011), no. 3, 1510-1531.

[27] H. Yu and P. Zhang, Global regularity to the 3D incompressible nematic liquid crystal flows with vacuum, Nonlinear Anal. 174 (2018), 209-222.

Yang Liu

College of Mathematics, Changchun Normal University, Changchun 130032, China and

Department of Mathematics, Nanjing University, Nanjing 210093, China

E-mail address: liuyang19850524@163.com 